Research Article

\title{
Explicit Exact Solutions of the (2+1)-Dimensional Integro-Differential Jaulent-Miodek Evolution Equation Using the Reliable Methods
}

\author{
Supaporn Kaewta $\mathbb{D}^{1},{ }^{1}$ Sekson Sirisubtawee $\mathbb{D}^{1,},{ }^{1,2}$ and Nattawut Khansai ${ }^{1}{ }^{1}$ \\ ${ }^{1}$ Department of Mathematics, Faculty of Applied Science, King Mongkut's University of Technology North Bangkok, \\ Bangkok 10800, Thailand \\ ${ }^{2}$ Centre of Excellence in Mathematics CHE, Si Ayutthaya Road Bangkok 10400, Thailand
}

Correspondence should be addressed to Sekson Sirisubtawee; sekson.s@sci.kmutnb.ac.th

Received 30 July 2019; Revised 23 December 2019; Accepted 3 January 2020; Published 27 April 2020

Academic Editor: Hans Engler

Copyright (c) 2020 Supaporn Kaewta et al. This is an open access article distributed under the Creative Commons Attribution License, which permits unrestricted use, distribution, and reproduction in any medium, provided the original work is properly cited.

\begin{abstract}
In this article, we utilize the $\left(G^{\prime} / G^{2}\right)$-expansion method and the Jacobi elliptic equation method to analytically solve the $(2+1)$ dimensional integro-differential Jaulent-Miodek equation for exact solutions. The equation is shortly called the Jaulent-Miodek equation, which was first derived by Jaulent and Miodek and associated with energy-dependent Schrödinger potentials (Jaulent and Miodek, 1976; Jaulent, 1976). The equation is converted into a fourth order partial differential equation using a transformation. After applying a traveling wave transformation to the resulting partial differential equation, we obtain an ordinary differential equation which is the main equation to which the both schemes are applied. As a first step, the two methods give us distinguish systems of algebraic equations. The first method provides exact traveling wave solutions including the logarithmic function solutions of trigonometric functions, hyperbolic functions, and polynomial functions. The second approach provides the Jacobi elliptic function solutions depending upon their modulus values. Some of the obtained solutions are graphically characterized by the distinct physical structures such as singular periodic traveling wave solutions and peakons. A comparison between our results and the ones obtained from the previous literature is given. Obtaining the exact solutions of the equation shows the simplicity, efficiency, and reliability of the used methods, which can be applied to other nonlinear partial differential equations taking place in mathematical physics.
\end{abstract}

\section{Introduction}

Nonlinear partial differential equations (NPDEs) are extensively used to explain complex phenomena in various fields of applied sciences, especially in physics and engineering. Nonlinear evolution equations, which are formulated using NPDEs, describe more than one of dispersion, dissipation, diffusion, reaction, and convection. The investigation of searching solutions for nonlinear evolution equations plays an important role in nonlinear physical science because the solutions can describe various natural phenomena of the problems such as vibrations, solitons, and propagation with a finite speed. There are two essential types of solutions for NPDEs, which are analytical and exact solutions. Some examples of the schemes used to obtain analytical approximate solutions to NPDEs are the homotopy analysis method (HAM) [3], the Adomian decomposition method (ADM) [4, 5], the modified Laplace variational iteration method (ML-VIM) [6], and the reduced differential transform method [7], while many effective methods have been proposed to obtain exact solutions of NPDEs including fractional order partial differential equations such as the generalized Kudryashov method [8-10], the amplitude ansatz method [11], the He's semiinverse method $[12,13]$, the exp-function method [14, 15], the auxiliary equation method $[16,17]$, the extended trial equation method $[18,19]$, and the extended direct algebraic method [20]. Moreover, the sine-cosine method [21, 22], the 
tanh-coth method $[23,24]$, the extended sech-tanh method [25], the sine-Gordon expansion method [26-28], and the $\left(G^{\prime} / G\right)$-expansion method [29-31] have been recently utilized to find analytical exact solutions of NPDEs as well. The advantage of finding exact solutions of nonlinear partial differential equations (NPDEs) is that they do not give any error terms for the problems which are better than numerical solutions of the problems.

The Jaulent-Miodek equation describes many branches of physics such as condensed matter physics, fluid dynamics, and optics [32]. In particular, the $(2+1)$-dimensional Jaulent-Miodek (JM) equation associates with energy-dependent Schrödinger potential [33]. The investigations of finding exact solutions of some kinds of the Jaulent-Miodek equations are as follows. In 2007, Wazwaz [34] used the tanh-coth and the sech methods to find exact solutions of the Jaulent-Miodek system. The obtained solutions included solitons, kink solutions, complex solutions, and solitary wave solutions. In 2008, He and Zhang [35] worked on the same system using the exp-function method. A generalized solitary wave solution and a generalized compacton-like solution were acquired. Jia-Min et al. [36] utilized the variational iteration method, which was combined with the Jacobian-function method, to solve the Jaulent-Miodek system. The resulting solutions consisted of doubly periodic wave solutions, solitary wave solutions, bell-type solitary wave solutions, and kink-type solitary wave solutions. In 2009, Wazwaz [37] used Hirota's bilinear method to solve the $(2+1)$-dimensional Jaulent-Miodek equation for its exact solutions. The multiple kink solutions and multiple singular kink solutions were constructed. In 2010, the $(1+1)$-dimensional Jaulent-Miodek equations were solved using the extended tanh method [38]. Lü et al. [39] obtained exact solutions of the coupled Jaulent-Miodek equations via the generalized $\left(G^{\prime} / G\right)$-expansion method. As a result, some new exact solutions were obtained including triangular periodic wave solutions, exponential solutions, and complex traveling solutions. In 2012, the $\left(G^{\prime} / G\right)$-expansion method was used to construct some new traveling wave solutions including hyperbolic function, trigonometric function, and rational function solutions of the $(2+1)$-dimensional Jaulent-Miodek equation [40]. Wazwaz [41] used the simplified form of Hirota's direct method to obtain multiple soliton solutions of the $(3+1)$-dimensional nonlinear models generated by the Jaulent-Miodek hierarchy. Zhang et al. [42] solved the $(2+1)$-dimensional Jaulent-Miodek equation using the direct symmetry method for the exact solutions including polynomial solutions, Airy function solutions, elliptic periodic solutions, and rational solutions. In 2015, Matinfar et al. [43] utilized the first integral method to obtain the kink-type and soliton solutions of the $(2+1)$ dimensional Jaulent-Miodek equation, while Li et al. [32] investigated the extended $(2+1)$-dimensional Jaulent-Miodek equation via Lie symmetries. In 2018, Gu et al. [44] derived exact traveling wave solutions of the $(2+1)$ dimensional Jaulent-Miodek equation using the complex method. Sadat and Kassem [45] solved the $(2+1)$-dimensional Jaulent-Miodek equation using the integrating factor technique. However, we here consider the $(2+1)$-dimensional integro-differential Jaulent-Miodek evolution equation $[37,42,44]$ :

$$
\begin{aligned}
& a_{1} W_{t}+a_{2} W^{2} W_{x}-W_{x x x}-a_{3} W_{x} \partial_{x}^{-1} W_{y}-a_{4} W W_{y} \\
& \quad+a_{5} \partial_{x}^{-1} W_{y y}=0,
\end{aligned}
$$

where $a_{1}, a_{2}, a_{3}, a_{4}, a_{5}$ are arbitrary constants and $\partial_{x}^{-1}=\int \mathrm{d} x$. Setting $W=u_{x}$ and substituting it into (1), we obtain the equivalent form of (1) as follows:

$$
a_{1} u_{x t}+a_{2} u_{x}^{2} u_{x x}-u_{x x x x}-a_{3} u_{x x} u_{y}-a_{4} u_{x} u_{x y}+a_{5} u_{y y}=0 .
$$

In this paper, we aim to use the $\left(G^{\prime} / G^{2}\right)$-expansion method [46-48] and the Jacobi elliptic equation method [49-52] to solve (2) for its exact traveling wave solutions.

The rest arrangement of the present paper is as follows. In Section 2, the algorithms of the $\left(G^{\prime} / G^{2}\right)$-expansion method and the Jacobi elliptic equation method are concisely given. In Section 3, the application of the methods for obtaining the exact solutions of (2) is demonstrated. We provide graphs and physical explanations of some selected exact solutions of the equation obtained using the two methods in Section 4. Finally, the conclusions are drawn in Section 5 .

\section{Description of the Methods}

In this section, we provide the concise description of the $\left(G^{\prime} / G^{2}\right)$-expansion method [46-48] and the Jacobi elliptic equation method [49-52]. Since both methods have a common initial step, then we will describe the preliminary step which is the conversion from a partial differential equation into an ordinary differential equation (ODE) using a traveling wave transformation. Consider a nonlinear evolution partial differential equation in three independent variables $t, x$, and $y$ as follows:

$$
F\left(u, u_{t}, u_{x}, u_{y}, u_{t t}, u_{x x}, u_{y y}, u_{t x}, u_{t y}, \ldots\right)=0,
$$

where $F$ is a polynomial of the unknown function $u=u(x, y, t)$ and its various partial derivatives in which the highest order derivatives and nonlinear terms are involved. Converting (3) into an ODE using the following traveling wave transformation

$$
\begin{aligned}
u(x, y, t) & =U(\xi), \\
\xi & =x+l y+\lambda t,
\end{aligned}
$$

where $l$ and $\lambda$ are nonzero arbitrary constants to be determined later and then integrating the resulting equation with respect to $\xi$ as many as possible; hence, (3) is reduced to the ODE in $U=U(\xi)$ as follows:

$$
P\left(U, U^{\prime}, U^{\prime \prime}, U^{\prime \prime \prime}, \ldots\right)=0,
$$

where $P$ is a polynomial of $U(\xi)$ and its various derivatives. The prime notation $\left({ }^{\prime}\right)$ denotes the derivative with respect to $\xi$. 
2.1. Algorithm of the $\left(G^{\prime} / G^{2}\right)$-Expansion Method. The main steps of the $\left(G^{\prime} / G^{2}\right)$-expansion method are as follows [46-48].

Step 1. Suppose that the formal solution of the ODE (5) can be written in powers of $\left(G^{\prime} / G^{2}\right)$ as follows:

$$
U(\xi)=\alpha_{0}+\sum_{j=1}^{N}\left[\alpha_{j}\left(\frac{G^{\prime}}{G^{2}}\right)^{j}+\beta_{j}\left(\frac{G^{\prime}}{G^{2}}\right)^{-j}\right]
$$

where $G=G(\xi)$ satisfies the following nonlinear ODE:

$$
\left(\frac{G^{\prime}}{G^{2}}\right)^{\prime}=\mu+\omega\left(\frac{G^{\prime}}{G^{2}}\right)^{2}
$$

in which $\mu \neq 1$ and $\omega \neq 0$ are integers. The unknown constants $\alpha_{N}$ or $\beta_{N}$ may be zero, but both of them cannot be zero simultaneously. The coefficients $\alpha_{0}, \alpha_{j}, \beta_{j}(j=1,2, \ldots, N)$ are unknown constants to be determined at a later step.

Step 2. The value of the positive integer $N$ can be calculated using the homogeneous balance principle, i.e., by balancing between the highest order derivatives and the nonlinear terms appearing in (5). More precisely, if the degree of $U(\xi)$ is $\operatorname{Deg}[U(\xi)]=N$, then the degree of the other terms will be expressed as follows:

$$
\begin{gathered}
\operatorname{Deg}\left[\frac{\mathrm{d}^{q} U(\xi)}{\mathrm{d} \xi^{q}}\right]=N+q, \\
\operatorname{Deg}\left[(U(\xi))^{p}\left(\frac{\mathrm{d}^{q} U(\xi)}{\mathrm{d} \xi^{q}}\right)^{s}\right]=N p+s(N+q) .
\end{gathered}
$$

Step 3. Substituting (6) along with (7) into (5), we obtain a polynomial in $\left(G^{\prime} / G^{2}\right)$. Collecting all coefficients of likepower of $\left(G^{\prime} / G^{2}\right)^{k}$ with $k=0, \pm 1, \pm 2, \ldots, \pm M$, where $M$ is some positive integer and then setting all of the obtained coefficients to zero, we obtain a system of nonlinear algebraic equations for the following unknown constants $\alpha_{0}, \alpha_{j}, \beta_{j}(j=1,2, \ldots, N), l$, and $\lambda$. Assume that the resulting algebraic system can be solved for the unknown constants using symbolic software packages such as Maple.

Step 4. The general solutions of (7) can be categorized into the following three cases when $A, B$ are arbitrary nonzero constants.

If $\mu \omega>0$, then we obtain the general solution:

$$
\frac{G^{\prime}}{G^{2}}=\sqrt{\frac{\mu}{\omega}}\left(\frac{A \cos (\sqrt{\mu \omega} \xi)+B \sin (\sqrt{\mu \omega} \xi)}{B \cos (\sqrt{\mu \omega} \xi)-A \sin (\sqrt{\mu \omega} \xi)}\right) .
$$

If $\mu \omega<0$, then we have the following general solution:

$$
\frac{G^{\prime}}{G^{2}}=\frac{1}{2 \omega}\left(2 \sqrt{|\mu \omega|}-\frac{4 A \sqrt{|\mu \omega|} e^{2 \xi \sqrt{|\mu \omega|}}}{A e^{2 \xi \sqrt{|\mu \omega|}}-B}\right),
$$

which is equivalent to

$$
\frac{G^{\prime}}{G^{2}}=-\frac{\sqrt{|\mu \omega|}}{\omega}\left(\frac{A \sinh (2 \sqrt{|\mu \omega|} \xi)+A \cosh (2 \sqrt{|\mu \omega|} \xi)+B}{A \sinh (2 \sqrt{|\mu \omega|} \xi)+A \cosh (2 \sqrt{|\mu \omega|} \xi)-B}\right) .
$$

If $\mu=0$ and $\omega \neq 0$, then the general solution of (7) is

$$
\frac{G^{\prime}}{G^{2}}=-\frac{A}{\omega(A \xi+B)} \text {. }
$$

The explicit exact solutions of (3) can be obtained by substituting the values of $\alpha_{0}, \alpha_{j}, \beta_{j}(j=1,2, \ldots, N), l, \lambda$ and the solutions (9)-(12) into (6) with the transformation (4).

2.2. Algorithm of the Jacobi Elliptic Equation Method. The primary steps of the Jacobi elliptic equation method [49-52] can be summarized as follows.

Step 1. We assume that the solution of (5) has the following form:

$$
U(\xi)=\sum_{i=-N}^{N} \alpha_{i}[\psi(\xi)]^{i}
$$

where $\alpha_{i}(i=-N, \ldots, N)$ are constants to be determined later, such that $\alpha_{N}^{2}+\alpha_{-N}^{2} \neq 0$, while the function $\psi(\xi)$ satisfies the Jacobi elliptic equation:

$$
\left[\psi^{\prime}(\xi)\right]^{2}=l_{0}+l_{2} \psi^{2}(\xi)+l_{4} \psi^{4}(\xi)
$$

where $l_{0}, l_{2}, l_{4}$ are real constants to be determined.

Step 2. We determine the positive integer $N$ in (13) by applying the homogeneous balance principle to (5). The balancing rules are already described in (8) of the previous method.

Step 3. Substituting (13) along with (14) into (5) and collecting all of the coefficients of $\left[\psi^{\prime}(\xi)\right]^{q}[\psi(\xi)]^{p}$, where $q=0,1$ and $p=0, \pm 1, \pm 2, \ldots, \pm P$, where $P$ is some positive integer, and then setting them to zero, we yield a system of algebraic equations, which can be solved using symbolic software packages such as Maple for the values of $\alpha_{i}(i=-N, \ldots, N), l_{0}, l_{2}, l_{4}, l$, and $\lambda$.

Step 4. It is well-known in [49-51] that (14) has the Jacobi elliptic function solutions as follows: 


\begin{tabular}{|c|c|c|c|c|}
\hline Case & $l_{0}$ & $l_{2}$ & $l_{4}$ & $\psi(\xi)$ \\
\hline 1 & 1 & $-\left(1+M^{2}\right)$ & $M^{2}$ & $\operatorname{sn}(\xi)$ or cd $(\xi)$ \\
\hline 2 & $1-M^{2}$ & $2 M^{2}-1$ & $-M^{2}$ & $\operatorname{cn}(\xi)$ \\
\hline 3 & $M^{2}-1$ & $2-M^{2}$ & -1 & $\operatorname{dn}(\xi)$ \\
\hline 4 & $M^{2}$ & $-\left(1+M^{2}\right)$ & 1 & $\mathrm{~ns}(\xi)$ or dc $(\xi)$ \\
\hline 5 & $-M^{2}$ & $2 M^{2}-1$ & $1-M^{2}$ & $\mathrm{nc}(\xi)$ \\
\hline 6 & -1 & $2-M^{2}$ & $M^{2}-1$ & $\operatorname{nd}(\xi)$ \\
\hline 7 & 1 & $2-M^{2}$ & $1-M^{2}$ & $\operatorname{sc}(\xi)$ \\
\hline 8 & 1 & $2 M^{2}-1$ & $-M^{2}\left(1-M^{2}\right)$ & $\operatorname{sd}(\xi)$ \\
\hline 9 & $1-M^{2}$ & $2-M^{2}$ & 1 & $\operatorname{cs}(\xi)$ \\
\hline 10 & $-M^{2}\left(1-M^{2}\right)$ & $2 M^{2}-1$ & 1 & $\mathrm{ds}(\xi)$ \\
\hline 11 & $\frac{1-M^{2}}{4}$ & $\frac{1+M^{2}}{2}$ & $\frac{1-M^{2}}{4}$ & $\mathrm{nc}(\xi) \pm \mathrm{sc}(\xi)$ \\
\hline 12 & $\frac{-\left(1-M^{2}\right)^{2}}{4}$ & $\frac{1+M^{2}}{2}$ & $-\frac{1}{4}$ & $M \operatorname{cn}(\xi) \pm \operatorname{dn}(\xi)$ \\
\hline 13 & $\frac{1}{4}$ & $\frac{1-2 M^{2}}{2}$ & $\frac{1}{4}$ & $\frac{\operatorname{sn}(\xi)}{1 \pm \operatorname{cn}(\xi)}$ \\
\hline 14 & $\frac{1-M^{2}}{4}$ & $\frac{1+M^{2}}{2}$ & $\frac{1-M^{2}}{4}$ & $\frac{\operatorname{cn}(\xi)}{1 \pm \operatorname{sn}(\xi)}$ \\
\hline 15 & $\frac{1}{4}$ & $\frac{1+M^{2}}{2}$ & $\frac{\left(1-M^{2}\right)^{2}}{4}$ & $\frac{\operatorname{sn}(\xi)}{\operatorname{cn}(\xi) \pm \operatorname{dn}(\xi)}$ \\
\hline
\end{tabular}

Remark 1. In the abovementioned table: $\operatorname{sn}(\xi)=\operatorname{sn}(\xi, M), \quad \operatorname{sd}(\xi)=1 / \mathrm{ds}(\xi)=\operatorname{sn}(\xi) / \operatorname{dn}(\xi), \quad \operatorname{dc}(\xi)=1 / \operatorname{cd}(\xi)=\operatorname{dn}(\xi) /$ $\operatorname{cn}(\xi)=\operatorname{cn}(\xi, M), \operatorname{dn}(\xi)=\operatorname{dn}(\xi, M), \operatorname{ns}(\xi)=\operatorname{ns}(\xi, M)$, $\mathrm{nc}(\xi)=\mathrm{nc}(\xi, M), \mathrm{nd}(\xi)=\operatorname{nd}(\xi, M), \quad \operatorname{cs}(\xi)=\operatorname{cs}(\xi, M)$, $\mathrm{ds}(\xi)=\mathrm{ds}(\xi, M), \quad \operatorname{cd}(\xi)=\mathrm{cd}(\xi, M), \quad \operatorname{sc}(\xi)=\operatorname{sc}(\xi, M)$, $\operatorname{sd}(\xi)=\operatorname{sd}(\xi, M)$, and $\mathrm{dc}(\xi)=\mathrm{dc}(\xi, M)$ are the Jacobi elliptic functions and $\operatorname{am}(\xi)=\operatorname{am}(\xi, M)$ is the Jacobi amplitude function. The constant $M$ with $0<M<1$ is the modulus of these functions.

Remark 2. It can be noticed that $\operatorname{sn}(\xi)=1 / \mathrm{ns}(\xi), \mathrm{cn}(\xi)=$ $1 / \operatorname{nc}(\xi), \quad \operatorname{dn}(\xi)=1 / \operatorname{nd}(\xi), \quad \operatorname{sc}(\xi)=1 / \operatorname{cs}(\xi)=\operatorname{sn}(\xi) / \mathrm{cn}(\xi)$,

$\operatorname{cn}(\xi)$.

Remark 3. The Jacobi elliptic functions can be transformed into hyperbolic functions when $M=1$ as follows: $\operatorname{sn}(\xi, 1)=$ $\tanh (\xi), \operatorname{cd}(\xi, 1)=1, \operatorname{cn}(\xi, 1)=\operatorname{sech}(\xi), \operatorname{dn}(\xi, 1)=\operatorname{sech}(\xi)$, $\operatorname{ns}(\xi, 1)=\operatorname{coth}(\xi), \quad \operatorname{cs}(\xi, 1)=\operatorname{csch}(\xi), \quad$ ds $(\xi, 1)=\operatorname{csch}(\xi)$, $\operatorname{sc}(\xi, 1)=\sinh (\xi), \quad \operatorname{sd}(\xi, 1)=\sinh (\xi), \quad \operatorname{nc}(\xi, 1)=\cosh (\xi)$, and into trigonometric functions when $M=0$ as follows: $\operatorname{sn}(\xi, 0)=\sin (\xi), \operatorname{cd}(\xi, 0)=\cos (\xi), \quad \operatorname{cn}(\xi, 0)=\cos (\xi)$, $\operatorname{dn}(\xi, 0)=1, \operatorname{ns}(\xi, 0)=\csc (\xi), \operatorname{cs}(\xi, 0)=\cot (\xi), \operatorname{ds}(\xi, 0)=$ $\csc (\xi), \operatorname{sc}(\xi, 0)=\tan (\xi), \operatorname{sd}(\xi, 0)=\sin (\xi), \operatorname{nc}(\xi, 0)=\sec (\xi)$. 
Remark 4. The Jacobi elliptic functions satisfy the following relations: $\quad \operatorname{sn}^{2}(\xi)+\mathrm{cn}^{2}(\xi)=1, \quad \mathrm{dn}^{2}(\xi)+M^{2} \mathrm{sn}^{2}(\xi)=1$, $\operatorname{sn}^{\prime}(\xi)=\operatorname{cn}(\xi) \operatorname{dn}(\xi), \quad \operatorname{cn}^{\prime}(\xi)=-\operatorname{sn}(\xi) \operatorname{dn}(\xi), \quad \operatorname{dn}^{\prime}(\xi)=$ $-M^{2} \operatorname{sn}(\xi) \operatorname{cn}(\xi), \quad \operatorname{cd}^{\prime}(\xi)=-\left(1-M^{2}\right) \operatorname{sd}(\xi) \operatorname{nd}(\xi), \operatorname{ns}^{\prime}(\xi)=$ $-\operatorname{cs}(\xi) \mathrm{ds}(\xi), \quad \mathrm{dc}^{\prime}(\xi)=\left(1-M^{2}\right) \mathrm{nc}(\xi) \mathrm{sc}(\xi), \quad \mathrm{cn}^{\prime}(\xi)=$ $\operatorname{sc}(\xi) \mathrm{dc}(\xi), \operatorname{nd}^{\prime}(\xi)=M^{2} \mathrm{~cd}(\xi) \operatorname{sd}(\xi), \operatorname{sc}^{\prime}(\xi)=\mathrm{dc}(\xi) \mathrm{nc}(\xi)$, $\operatorname{cs}^{\prime}(\xi)=-\mathrm{ns}(\xi) \mathrm{ds}(\xi), \mathrm{ds}^{\prime}(\xi)=-\mathrm{cs}(\xi) \mathrm{ns}(\xi), \mathrm{sd}^{\prime}(\xi)=\mathrm{nd}(\xi)$ $\operatorname{cd}(\xi), \operatorname{am}^{\prime}(\xi)=\operatorname{dn}(\xi)$, where the prime notation $\left(^{\prime}\right)$ denotes the derivative with respect to $\xi$.

Step 5. Substituting the values of $\alpha_{i}(i=-N, \ldots, N)$, $l_{0}, l_{2}, l_{4}, l, \lambda$, and the Jacobi elliptic functions $\psi(\xi)$ with $\xi$ in (4) into (13), we finally obtain the exact solutions of (3).

\section{Application of the Methods}

Substituting traveling wave transform

$$
u(x, y, t)=v(\xi), \xi=x+l y+\lambda t,
$$

into (2) and then integrating it, we obtain

$$
v^{\prime \prime \prime}-\left(a_{1} \lambda+a_{5} l^{2}\right) v^{\prime}+\frac{l\left(a_{3}+a_{4}\right)}{2}\left(v^{\prime}\right)^{2}-\frac{a_{2}}{3}\left(v^{\prime}\right)^{3}-\delta=0,
$$

where $l$ and $\lambda$ are constants to be determined, and $\delta$ is a constant of integration. Setting $z=v^{\prime}$, (17) becomes

$$
z^{\prime \prime}-\left(a_{1} \lambda+a_{5} l^{2}\right) z+\frac{l\left(a_{3}+a_{4}\right)}{2} z^{2}-\frac{a_{2}}{3} z^{3}-\delta=0 .
$$

3.1. On Solving (18) Using the $\left(G^{\prime} / G^{2}\right)$-Expansion Method. Following the steps of the $\left(G^{\prime} / G^{2}\right)$-expansion method described in Section 2.1, we assume that the exact solution of (18) has the form

$$
z(\xi)=\alpha_{0}+\sum_{j=1}^{N}\left[\alpha_{j}\left(\frac{G^{\prime}}{G^{2}}\right)^{j}+\beta_{j}\left(\frac{G^{\prime}}{G^{2}}\right)^{-j}\right],
$$

where $G=G(\xi)$ satisfies (7). Using (8) to balance the terms $z^{\prime \prime}$ and $z^{3}$ in (18), we obtain $\operatorname{Deg}\left[z^{\prime \prime}\right]=N+2=\operatorname{Deg}\left[z^{3}\right]=$ $3 N$, leading to $N=1$. Hence, the solution can be written as

$$
z(\xi)=\alpha_{0}+\alpha_{1}\left(\frac{G^{\prime}}{G^{2}}\right)+\beta_{1}\left(\frac{G^{\prime}}{G^{2}}\right)^{-1}
$$

where $\alpha_{0}, \alpha_{1}, \beta_{1}$ are unknown constants with $\alpha_{1}, \beta_{1} \neq 0$. Substituting (20) into (18) along with (7), collecting all of the coefficients with the same power of $\left(G^{\prime} / G^{2}\right)$, and then setting these resulting coefficients to zero, we consequently obtain the following system of algebraic equations in $\alpha_{0}, \alpha_{1}, \beta_{1}$, $\mu, \omega, \lambda, l, \delta$ :

$$
\begin{aligned}
& \left(\frac{G^{\prime}}{G^{2}}\right)^{-3}: 2 \beta_{1} \mu^{2}-\frac{a_{2} \beta_{1}^{3}}{3}=0, \\
& \left(\frac{G^{\prime}}{G^{2}}\right)^{-2}: \frac{l\left(a_{3}+a_{4}\right) \beta_{1}^{2}}{2}-a_{2} \alpha_{0} \beta_{1}^{2}=0, \\
& \left(\frac{G^{\prime}}{G^{2}}\right)^{-1}:\left(a_{3}+a_{4}\right) l \alpha_{0} \beta_{1}-l^{2} a_{5} \beta_{1}-a_{2} \alpha_{0}^{2} \beta_{1}-a_{2} \alpha_{1} \beta_{1}^{2} \\
& -\lambda a_{1} \beta_{1}+2 \mu \omega \beta_{1}=0, \\
& \left(\frac{G^{\prime}}{G^{2}}\right)^{0}: \frac{\left(a_{3}+a_{4}\right) l \alpha_{0}^{2}}{2}-l^{2} a_{5} \alpha_{0}-\lambda a_{1} \alpha_{0}+\left(a_{3}+a_{4}\right) l \alpha_{1} \beta_{1} \\
& -2 a_{2} \alpha_{0} \alpha_{1} \beta_{1}-\delta-\frac{a_{2} \alpha_{0}^{3}}{3}=0, \\
& \left(\frac{G^{\prime}}{G^{2}}\right)^{1}:\left(a_{3}+a_{4}\right) l \alpha_{0} \alpha_{1}-l^{2} a_{5} \alpha_{1}-a_{2} \alpha_{0}^{2} \alpha_{1}-a_{2} \alpha_{1}^{2} \beta_{1} \\
& -\lambda a_{1} \alpha_{1}+2 \mu \omega \alpha_{1}=0, \\
& \left(\frac{G^{\prime}}{G^{2}}\right)^{2}: \frac{l\left(a_{3}+a_{4}\right) \alpha_{1}^{2}}{2}-a_{2} \alpha_{0} \alpha_{1}^{2}=0, \\
& \left(\frac{G^{\prime}}{G^{2}}\right)^{3}: 2 \alpha_{1} \omega^{2}-\frac{a_{2} \alpha_{1}^{3}}{3}=0 .
\end{aligned}
$$

Solving the obtained algebraic system (21) with the assistance of the Maple package program, we get the following three results.

Result 1

$$
\begin{aligned}
& \alpha_{0}=\frac{\left(a_{3}+a_{4}\right) l}{2 a_{2}}, \\
& \alpha_{1}= \pm \sqrt{\frac{6}{a_{2}} \omega} \\
& \beta_{1}=0, \\
& \delta=-\frac{\left(a_{3}+a_{4}\right)\left(\left(a_{3}+a_{4}\right)^{2} l^{2}+24 \mu \omega a_{2}\right) l}{24 a_{2}^{2}}, \\
& \lambda=\frac{\left(a_{3}+a_{4}\right)^{2} l^{2}-4 l^{2} a_{5} a_{2}+8 \mu \omega a_{2}}{4 a_{1} a_{2}},
\end{aligned}
$$

where $a_{1}, a_{2}, a_{3}, a_{4}, a_{5}, l, \omega$, and $\mu$ are arbitrary constants. In order to obtain the solution $v(\xi)$ of (17), we must integrate the solution $z(\xi)$ of (18) once. Then, the exact solution $u(x, y, t)$ of (2) can be obtained by replacing $\xi=x+l y+\lambda t$, where $\lambda$ is expressed in (22), into the solution $v(\xi)$. 
If $\mu \omega>0$, then the trigonometric function solution of (2), which is obtained by substituting the parameter values in (22) and the term $\left(G^{\prime} / G^{2}\right)$ in (9) into $(20)$, can be written as $u_{2}^{1}(x, y, t)=\frac{\left(a_{3}+a_{4}\right) l \xi}{2 a_{2}} \mp \sqrt{\frac{6}{a_{2}}} \ln (B \cos (\sqrt{\mu \omega} \xi)-A \sin (\sqrt{\mu \omega} \xi))$.

If $\mu \omega<0$, then the exponential function solution of (2), which is obtained by substituting the parameter values in (22) and the term $\left(G^{\prime} / G^{2}\right)$ in (10) into (20), can be written as $u_{2}^{1}(x, y, t)=\frac{\left(a_{3}+a_{4}\right) l \xi}{2 a_{2}} \pm \sqrt{\frac{6}{a_{2}}}\left(\sqrt{|\mu \omega|} \xi-\ln \left(A \mathrm{e}^{2 \sqrt{|\mu \omega|} \xi}-B\right)\right)$,

which is equivalent to

$$
\begin{aligned}
u_{2}^{1}(x, y, t)= & \frac{\left(a_{3}+a_{4}\right) l \xi}{2 a_{2}} \mp\left[\sqrt{\frac{6}{a_{2}}} \ln ((A+B) \tanh (\sqrt{|\mu \omega|} \xi)\right. \\
& \left.+A-B)-\sqrt{\frac{3}{2 a_{2}}} \ln \left(\tanh ^{2}(\sqrt{|\mu \omega|} \xi)-1\right)\right] .
\end{aligned}
$$

If $\mu=0, \omega \neq 0$, then the rational function solution of (2), which is obtained by substituting the parameter values in (22) and the term $\left(G^{\prime} / G^{2}\right)$ in (12) into (20), can be expressed as

$$
u_{3}^{1}(x, y, t)=\frac{\left(a_{3}+a_{4}\right) l \xi}{2 a_{2}} \mp \sqrt{\frac{6}{a_{2}}} \ln (A \xi+B) .
$$

Result 2

$$
\begin{aligned}
& \alpha_{0}=\frac{\left(a_{3}+a_{4}\right) l}{2 a_{2}}, \\
& \alpha_{1}=0, \\
& \beta_{1}= \pm \sqrt{\frac{6}{a_{2}} \mu,} \\
& \delta=-\frac{\left(a_{3}+a_{4}\right)\left(\left(a_{3}+a_{4}\right)^{2} l^{2}+24 \mu \omega a_{2}\right) l}{24 a_{2}^{2}}, \\
& \lambda=\frac{\left(a_{3}+a_{4}\right)^{2} l^{2}-4 l^{2} a_{5} a_{2}+8 \mu \omega a_{2}}{4 a_{1} a_{2}},
\end{aligned}
$$

where $a_{1}, a_{2}, a_{3}, a_{4}, a_{5}, l, \omega$ and $\mu$ are arbitrary constants. In order for obtain the solution $v(\xi)$ of (17), we must integrate the solution $z(\xi)$ of (18) once. Then, the exact solution $u(x, y, t)$ of (2) can be obtained by replacing $\xi=x+l y+\lambda t$ with $\lambda$ expressed in (27) into the solution $v(\xi)$.

If $\mu \omega>0$, then the trigonometric function solution of (2), which is constructed by substituting the parameter values in (27) and the term $\left(G^{\prime} / G^{2}\right)$ in (9) into (20), can be expressed as

$$
u_{1}^{2}(x, y, t)=\frac{\left(a_{3}+a_{4}\right) l \xi}{2 a_{2}} \pm \sqrt{\frac{6}{a_{2}}} \ln (A \cos (\sqrt{\mu \omega} \xi)+B \sin (\sqrt{\mu \omega} \xi))
$$

If $\mu \omega<0$, the exponential function solution of (2), which is generated by substituting the parameter values in (27) and the term $\left(G^{\prime} / G^{2}\right)$ in (10) into (20), can be written as

$$
u_{2}^{2}(x, y, t)=\frac{\left(a_{3}+a_{4}\right) l \xi}{2 a_{2}} \pm \sqrt{\frac{6}{a_{2}}}\left(\ln \left(A \mathrm{e}^{2 \sqrt{|\mu \omega|} \xi}+B\right)-\sqrt{|\mu \omega|} \xi\right)
$$

which is equivalent to

$$
\begin{aligned}
u_{2}^{2}(x, y, t)= & \frac{\left(a_{3}+a_{4}\right) l \xi}{2 a_{2}} \pm\left[\sqrt{\frac{6}{a_{2}}} \ln ((A-B) \tanh (\sqrt{|\mu \omega|} \xi)\right. \\
& \left.+A-B)-\sqrt{\frac{3}{2 a_{2}}} \ln \left(\tanh ^{2}(\sqrt{|\mu \omega|} \xi)-1\right)\right] .
\end{aligned}
$$

If $\mu=0, \omega \neq 0$, then the rational function solution of (2), which is obtained by substituting the parameter values in (27) and the term $\left(G^{\prime} / G^{2}\right)$ in (12) into (20), can be expressed as

$$
u_{3}^{2}(x, y, t)=\frac{\left(a_{3}+a_{4}\right) l \xi}{2 a_{2}}
$$

\section{Result 3}

Result 3.1

$$
\begin{aligned}
\alpha_{0} & =\frac{\left(a_{3}+a_{4}\right) l}{2 a_{2}}, \\
\alpha_{1} & =\sqrt{\frac{6}{a_{2}} \omega,} \\
\beta_{1} & = \pm \sqrt{\frac{6}{a_{2}} \mu,} \\
\delta & =\frac{\left(a_{3}+a_{4}\right)\left(12 a_{2}^{2} \alpha_{1} \beta_{1}-\left(a_{3}+a_{4}\right)^{2} l^{2}-24 \mu \omega a_{2}\right)}{24 a_{2}^{2}}, \\
\lambda & =-\frac{4 a_{2}^{2} \alpha_{1} \beta_{1}-\left(a_{3}+a_{4}\right)^{2} l^{2}+4 l^{2} a_{2} a_{5}-8 \mu \omega a_{2}}{4 a_{1} a_{2}},
\end{aligned}
$$

where $a_{1}, a_{2}, a_{3}, a_{4}, a_{5}, l, \omega$, and $\mu$ are arbitrary constants. In order to acquire the solution $v(\xi)$ of (17), we must integrate the solution $z(\xi)$ of (18) one time. Then, the exact solution $u(x, y, t)$ of (2) can be obtained by replacing $\xi=x+l y+\lambda t$, where $\lambda$ is expressed in (32), into the solution $v(\xi)$.

If $\mu \omega>0$, then the trigonometric function solution of (2), which is obtained by substituting the parameter values in (32) and the term $\left(G^{\prime} / G^{2}\right)$ in (9) into (20), can be written as 


$$
\begin{aligned}
u_{1}^{3}(x, y, t)= & \frac{\left(a_{3}+a_{4}\right) l \xi}{2 a_{2}}-\sqrt{\frac{6}{a_{2}}} \ln (B \cos (\sqrt{\mu \omega} \xi)-A \sin (\sqrt{\mu \omega} \xi)) \\
& \pm \sqrt{\frac{6}{a_{2}}} \ln (A \cos (\sqrt{\mu \omega} \xi)+B \sin (\sqrt{\mu \omega} \xi)) .
\end{aligned}
$$

If $\mu \omega<0$, then the exponential function solution of (2), which is attained by substituting the parameter values in (32) and the term $\left(G^{\prime} / G^{2}\right)$ in (10) into (20), can be expressed as

$$
\begin{aligned}
u_{2}^{3}(x, y, t)= & \frac{\left(a_{3}+a_{4}\right) l \xi}{2 a_{2}}+\sqrt{\frac{6}{a_{2}}}\left(\sqrt{|\mu \omega|} \xi-\ln \left(A \mathrm{e}^{2 \sqrt{|\mu \omega|} \xi}-B\right)\right) \\
& \mp \sqrt{\frac{6}{a_{2}}}\left(\sqrt{|\mu \omega|} \xi-\ln \left(A \mathrm{e}^{2 \sqrt{|\mu \omega|} \xi}+B\right)\right),
\end{aligned}
$$

or equivalently,

$$
\begin{aligned}
u_{2}^{3}(x, y, t)= & \frac{\left(a_{3}+a_{4}\right) l \xi}{2 a_{2}}-\sqrt{\frac{6}{a_{2}}} \ln ((A+B) \tanh (\sqrt{|\mu \omega|} \xi)+A-B) \\
& \pm \sqrt{\frac{6}{a_{2}}} \ln ((A-B) \tanh (\sqrt{|\mu \omega|} \xi)+A+B)
\end{aligned}
$$

If $\mu=0, \omega \neq 0$, then we get the solution (26).

\section{Result 3.2}

$$
\begin{aligned}
& \alpha_{0}=\frac{\left(a_{3}+a_{4}\right) l}{2 a_{2}}, \\
& \alpha_{1}=-\sqrt{\frac{6}{a_{2}} \omega,} \\
& \beta_{1}= \pm \sqrt{\frac{6}{a_{2}} \mu,} \\
& \delta=\frac{\left(a_{3}+a_{4}\right)\left(12 a_{2}^{2} \alpha_{1} \beta_{1}-\left(a_{3}+a_{4}\right)^{2} l^{2}-24 \mu \omega a_{2}\right) l}{24 a_{2}^{2}}, \\
& \lambda=-\frac{4 a_{2}^{2} \alpha_{1} \beta_{1}-\left(a_{3}+a_{4}\right)^{2} l^{2}+4 l^{2} a_{2} a_{5}-8 \mu \omega a_{2}}{4 a_{1} a_{2}},
\end{aligned}
$$

where $a_{1}, a_{2}, a_{3}, a_{4}, a_{5}, l, \omega$, and $\mu$ are arbitrary constants. In the same manner as mentioned above, we can obtain other solutions of (2) using the parameter values in (36). However, they are omitted here because of the minimalism.

3.2. On Solving (18) Using the Jacobi Elliptic Function Method. Balancing the terms $z^{3}$ and $z^{\prime \prime}$ in (18), we thus obtain the same balance number $N$ as the previous method, i.e., $N=1$. From (13), the formal solution of (18) has the form

$$
z(\xi)=\alpha_{0}+\alpha_{1} \psi(\xi)+\alpha_{-1} \psi^{-1}(\xi),
$$

where $\psi(\xi)$ satisfies (14), while $\alpha_{i}(i=0, \pm 1)$ are arbitrary constants to be determined later such that $\alpha_{1} \neq 0$ and $\alpha_{-1} \neq 0$. Substituting (37) into (18) along with (14), gathering all of the coefficients of $\left[\psi^{\prime}(\xi)\right]^{q}[\psi(\xi)]^{p}$ with $q=0,1$ and $p=0, \pm 1, \pm 2, \pm 3$, and then letting these resulting coefficients be zero, we consequently obtain the following algebraic equations in $\alpha_{0}, \alpha_{1}, \alpha_{-1}, l_{0}, l_{2}, l_{4}, l$, and $\lambda$ :

$$
\begin{aligned}
\psi(\xi)^{-3}: 2 \alpha_{-1} l_{0}-\frac{a_{2} \alpha_{-1}^{3}}{3}=0 & \\
\psi(\xi)^{-2}: & \frac{l\left(a_{3}+a_{4}\right) \alpha_{-1}^{2}}{2}-a_{2} \alpha_{0} \alpha_{-1}^{2}=0 \\
\psi(\xi)^{-1}: \quad & \left(a_{3}+a_{4}\right) l \alpha_{-1} \alpha_{0}-l^{2} a_{5} \alpha_{-1}-a_{2} \alpha_{-1}^{2} \alpha_{1}-a_{2} \alpha_{-1} \alpha_{0}^{2} \\
& -\lambda a_{1} \alpha_{-1}+\alpha_{-1} l_{2}=0 \\
\psi(\xi)^{0}: & l\left(a_{3}+a_{4}\right) \alpha_{1} \alpha_{-1}-2 a_{2} \alpha_{0} \alpha_{1} \alpha_{-1}-\alpha_{0} a_{1} \lambda-\alpha_{0} a_{5} l^{2} \\
& +\frac{l\left(a_{3}+a_{4}\right) \alpha_{0}^{2}}{2}-\delta-\frac{a_{2} \alpha_{0}^{3}}{3}=0 \\
\psi(\xi)^{1}: \quad & \left(a_{3}+a_{4}\right) l \alpha_{0} \alpha_{1}-l^{2} a_{5} \alpha_{1}-a_{2} \alpha_{-1} \alpha_{1}^{2}-a_{2} \alpha_{0}^{2} \alpha_{1} \\
& -\lambda a_{1} \alpha_{1}+\alpha_{1} l_{2}=0 \\
& l\left(a_{3}+a_{4}\right) \alpha_{1}^{2} \\
2 & \\
\psi(\xi)^{2}: a_{2} \alpha_{0} \alpha_{1}^{2}=0 & -\frac{a_{2} \alpha_{1}^{3}}{3}+2 \alpha_{1} l_{4}=0 \\
(\xi)^{3}: &
\end{aligned}
$$

Solving (38) with the aid of the Maple software package, we have three results of the parameter values as follows.

Result 4

$$
\begin{aligned}
\alpha_{0} & =\frac{\left(a_{3}+a_{4}\right) l}{2 a_{2}}, \\
\alpha_{1} & =0, \\
\alpha_{-1} & = \pm \sqrt{\frac{6 l_{0}}{a_{2}}}, \\
\lambda & =\frac{\left(a_{3}+a_{4}\right)^{2} l^{2}-4 l^{2} a_{5} a_{2}+4 l_{2} a_{2}}{4 a_{1} a_{2}}, \\
\delta & =-\frac{l\left(a_{3}+a_{4}\right)\left(\left(a_{3}+a_{4}\right)^{2} l^{2}+12 l_{2} a_{2}\right)}{24 a_{2}^{2}},
\end{aligned}
$$

where $a_{1}, a_{2}, a_{3}, a_{4}, a_{5}, l_{0}, l_{2}, l_{4}$, and $l$ are arbitrary constants, provided that $a_{1} a_{2} \neq 0, l_{0} a_{2}>0$. Using the obtained results (39), we have the following cases of the exact solutions for (2). 
Case 1. When $l_{0}=1, l_{2}=-\left(1+M^{2}\right)$ and $l_{4}=M^{2}$, we have

$$
\begin{aligned}
& u_{1,1}^{1}(x, y, t)=\frac{\left(a_{3}+a_{4}\right) l \xi}{2 a_{2}} \pm \sqrt{\frac{6}{a_{2}}} \ln \left(\frac{\operatorname{sn}(\xi)}{\operatorname{cn}(\xi)+\operatorname{dn}(\xi)}\right) \\
& u_{1,2}^{1}(x, y, t)=\frac{\left(a_{3}+a_{4}\right) l \xi}{2 a_{2}} \pm \sqrt{\frac{6}{a_{2}}} \ln \left(\frac{\operatorname{sd}(\xi)+\operatorname{nd}(\xi)}{\operatorname{cd}(\xi)}\right),
\end{aligned}
$$

where $\xi=x+l y-\left(\left(4 l^{2} a_{5} a_{2}-l^{2} a_{3}^{2}-2 l^{2} a_{3} a_{4}-l^{2} a_{4}^{2}+4 a_{2}\left(M^{2}\right.\right.\right.$ $\left.+1)) / 4 a_{1} a_{2}\right) t$ and $a_{2}>0$.

If $M=0$, then (40) and (41) become

$$
u_{1,1}^{1}(x, y, t)=\frac{\left(a_{3}+a_{4}\right) l \xi}{2 a_{2}} \pm \sqrt{\frac{6}{a_{2}}} \ln (\csc (\xi)-\cot (\xi)) \text {, }
$$

$$
u_{1,2}^{1}(x, y, t)=\frac{\left(a_{3}+a_{4}\right) l \xi}{2 a_{2}} \pm \sqrt{\frac{6}{a_{2}}} \ln (\sec (\xi)+\tan (\xi)),
$$

respectively, where $\xi=x+l y-\left(\left(4 l^{2} a_{2} a_{5}-l^{2} a_{3}^{2}-2 l^{2} a_{3} a_{4}-\right.\right.$ $\left.\left.l^{2} a_{4}^{2}+4 a_{2}\right) / 4 a_{1} a_{2}\right) t$ and $a_{2}>0$.

If $M=1$, then (40) and (41) turn out to be only one solution as follows:

$$
u_{1,1}^{1}(x, y, t)=\left(\frac{\left(a_{3}+a_{4}\right) l}{2 a_{2}} \pm \sqrt{\frac{6}{a_{2}}}\right) \xi,
$$

where $\xi=x+l y-\left(\left(4 l^{2} a_{2} a_{5}-l^{2} a_{3}^{2}-2 l^{2} a_{3} a_{4}-l^{2} a_{4}^{2}+8 a_{2}\right) /\right.$ $\left.4 a_{1} a_{2}\right) t$ and $a_{2}>0$.

Case 2. When $l_{0}=1-M^{2}, l_{2}=2 M^{2}-1$ and $l_{4}=-M^{2}$, we have

$$
u_{2,1}^{1}(x, y, t)=\frac{\left(a_{3}+a_{4}\right) l \xi}{2 a_{2}} \pm \sqrt{\frac{6}{a_{2}}} \ln \left(\frac{\sqrt{1-M^{2}} \operatorname{sn}(\xi)+\operatorname{dn}(\xi)}{\operatorname{cn}(\xi)}\right)
$$

where $\xi=x+l y-\left(\left(4 l^{2} a_{2} a_{5}-l^{2} a_{3}^{2}-2 l^{2} a_{3} a_{4}-l^{2} a_{4}^{2}-4 a_{2}\right)\right.$. $\left.\left(2 M^{2}-1\right)\right) / 4 a_{1} a_{2} t$ and $a_{2}>0$.

If $M=0$, then (45) becomes the solution (43) with $\xi=$ $x+l y-\left(\left(4 l^{2} a_{2} a_{5}-l^{2} a_{3}^{2}-2 l^{2} a_{3} a_{4}-l^{2} a_{4}^{2}+4 a_{2}\right) / 4 a_{1} a_{2}\right) t$.

If $M=1$, then (45) reduces to

$$
u_{2,1}^{1}(x, y, t)=\frac{\left(a_{3}+a_{4}\right) l \xi}{2 a_{2}},
$$

where $\xi=x+l y-\left(\left(4 l^{2} a_{2} a_{5}-l^{2} a_{3}^{2}-2 l^{2} a_{3} a_{4}-l^{2} a_{4}^{2}-4 a_{2}\right) /\right.$ $\left.4 a_{1} a_{2}\right) t$.

Case 3. When $l_{0}=M^{2}-1, l_{2}=2-M^{2}, l_{4}=-1$, we have $u_{3,1}^{1}(x, y, t)=\frac{\left(a_{3}+a_{4}\right) l \xi}{2 a_{2}} \pm \sqrt{-\frac{6}{a_{2}}} \arctan \left(\frac{\sqrt{1-M^{2}} \operatorname{sn}(\xi)}{\mathrm{cn}(\xi)}\right)$, where $\quad \xi=x+l y-\left(\left(4 l^{2} a_{2} a_{5}-l^{2} a_{3}^{2}-2 l^{2} a_{3} a_{4}-l^{2} a_{4}^{2}-4 a_{2}\right.\right.$ $\left.\left.\cdot\left(2-M^{2}\right)\right) / 4 a_{1} a_{2}\right) t$ and $a_{2}<0$.

If $M=0$, then (47) turns out to be

$$
u_{3,1}^{1}(x, y, t)=\left(\frac{\left(a_{3}+a_{4}\right) l}{2 a_{2}} \pm \sqrt{-\frac{6}{a_{2}}}\right) \xi,
$$

where $\xi=x+l y-\left(\left(4 l^{2} a_{2} a_{5}-l^{2} a_{3}^{2}-2 l^{2} a_{3} a_{4}-l^{2} a_{4}^{2}-8 a_{2}\right) /\right.$ $\left.4 a_{1} a_{2}\right) t$ and $a_{2}<0$.

If $M=1$, then (47) reduces to the solution (46) with $\xi=x+l y-\left(1 / 4 a_{1} a_{2}\right)\left(4 l^{2} a_{2} a_{5}-l^{2} a_{3}^{2}-2 l^{2} a_{3} a_{4}-l^{2} a_{4}^{2}-\right.$ $\left.4 a_{2}-4 a_{2} 4 a_{1} a_{2}\right) t$.

Case 4. When $l_{0}=M^{2}, l_{2}=-\left(1+M^{2}\right), l_{4}=1$, we have

$$
u_{4,1}^{1}(x, y, t)=\frac{\left(a_{3}+a_{4}\right) l \xi}{2 a_{2}} \pm \sqrt{\frac{6}{a_{2}}} \ln \left(\frac{\mathrm{ds}(\xi)-M \operatorname{cs}(\xi)}{\mathrm{ns}(\xi)}\right),
$$

$$
u_{4,2}^{1}(x, y, t)=\frac{\left(a_{3}+a_{4}\right) l \xi}{2 a_{2}} \pm \sqrt{\frac{6}{a_{2}}} \ln \left(\frac{\mathrm{nc}(\xi)+M s c(\xi)}{\mathrm{dc}(\xi)}\right),
$$

where $\xi=x+l y-\left(\left(4 l^{2} a_{2} a_{5}-l^{2} a_{3}^{2}-2 l^{2} a_{3} a_{4}-l^{2} a_{4}^{2}+4 a_{2}\right.\right.$. $\left.\left.\left(M^{2}+1\right)\right) / 4 a_{1} a_{2}\right) t$ and $a_{2}>0$.

If $M=0$, then both (49) and (50) reduce to the solution (46) with $\quad \xi=x+l y-\left(\left(4 l^{2} a_{2} a_{5}-l^{2} a_{3}^{2}-2 l^{2} a_{3} a_{4}\right.\right.$ $\left.\left.-l^{2} a_{4}^{2}+4 a_{2}\right) / 4 a_{1} a_{2}\right) t$.

If $M=1$, then both (49) and (50) turn out to be

$$
\begin{aligned}
u_{4,1}^{1}(x, y, t)= & \frac{\left(a_{3}+a_{4}\right) l \xi}{2 a_{2}} \pm \sqrt{\frac{6}{a_{2}}} \ln (\cosh (\xi)) \\
& \mp \frac{1}{2} \sqrt{\frac{6}{a_{2}}}(\ln (\cosh (\xi)-1)+\ln (\cosh (\xi)+1)),
\end{aligned}
$$

where

$\xi=x+l y-\left(\left(4 l^{2} a_{2} a_{5}-l^{2} a_{3}^{2}-2 l^{2} a_{3} a_{4}-l^{2} a_{4}^{2}+8 a_{2}\right) /\right.$

$\left.4 a_{1} a_{2}\right) t$ and $a_{2}>0$.

Case 5. When $l_{0}=-M^{2}, l_{2}=2 M^{2}-1, l_{4}=1-M^{2}$, we obtain

$$
u_{5,1}^{1}(x, y, t)=\frac{\left(a_{3}+a_{4}\right) l \xi}{2 a_{2}} \pm \sqrt{-\frac{6}{a_{2}}} \arctan (M \operatorname{sd}(\xi)),
$$

where $\xi=x+l y-\left(\left(4 l^{2} a_{2} a_{5}-l^{2} a_{3}^{2}-2 l^{2} a_{3} a_{4}-l^{2} a_{4}^{2}-4 a_{2}\right.\right.$. $\left.\left.\left(2 M^{2}-1\right)\right) / 4 a_{1} a_{2}\right) t$ and $a_{2}<0$.

If $M=0$, then (52) reduces to the solution (46) with $\xi=$ $x+l y-\left(1 / 4 a_{1} a_{2}\right)\left(4 l^{2} a_{2} a_{5}-l^{2} a_{3}^{2}-2 l^{2} a_{3} a_{4}-l^{2} a_{4}^{2}+4 a_{2}\right) t$.

If $M=1$, then (52) becomes

$$
u_{5,1}^{1}(x, y, t)=\frac{\left(a_{3}+a_{4}\right) l \xi}{2 a_{2}} \pm 2 \sqrt{-\frac{6}{a_{2}}} \arctan \left(\mathrm{e}^{\xi}\right),
$$

where $\xi=x+l y-\left(\left(4 l^{2} a_{2} a_{5}-l^{2} a_{3}^{2}-2 l^{2} a_{3} a_{4}-l^{2} a_{4}^{2}-4 a_{2}\right) /\right.$ $\left.4 a_{1} a_{2}\right) t$ and $a_{2}<0$. 
Case 6. When $l_{0}=-1, l_{2}=2-M^{2}, l_{4}=M^{2}-1$, then we have

$$
u_{6,1}^{1}(x, y, t)=\frac{\left(a_{3}+a_{4}\right) l \xi}{2 a_{2}} \pm \sqrt{-\frac{6}{a_{2}}} \operatorname{am}(\xi),
$$

where $\xi=x+l y-\left(\left(4 l^{2} a_{2} a_{5}-l^{2} a_{3}^{2}-2 l^{2} a_{3} a_{4}-l^{2} a_{4}^{2}-4 a_{2}\right.\right.$. $\left.\left.\left(2-M^{2}\right)\right) / 4 a_{1} a_{2}\right) t$ and $a_{2}<0$.

If $M=0$, then (54) becomes the solution (48) with $\xi=$ $x+l y-\left(\left(4 l^{2} a_{2} a_{5}-l^{2} a_{3}^{2}-2 l^{2} a_{3} a_{4}-l^{2} a_{4}^{2}-8 a_{2}\right) / 4 a_{1} a_{2}\right) t$.

If $M=1$, then (54) turns out to be the solution (53) with $\xi=x+l y-\left(1 / 4 a_{1} a_{2}\right)\left(4 l^{2} a_{2} a_{5}-l^{2} a_{3}^{2}-2 l^{2} a_{3} a_{4}-l^{2} a_{4}^{2}-\right.$ $\left.4 a_{2}\right) t$.

Case 7. When $l_{0}=1, l_{2}=2-M^{2}, l_{4}=1-M^{2}$, then we have

$$
u_{7,1}^{1}(x, y, t)=\frac{\left(a_{3}+a_{4}\right) l \xi}{2 a_{2}} \pm \sqrt{\frac{6}{a_{2}} \ln \left(\frac{\mathrm{nc}(\xi)-\mathrm{dc}(\xi)}{\mathrm{sc}(\xi)}\right)},
$$

where $\xi=x+l y-\left(\left(4 l^{2} a_{2} a_{5}-l^{2} a_{3}^{2}-2 l^{2} a_{3} a_{4}-l^{2} a_{4}^{2}-4 a_{2}\right.\right.$. $\left.\left.\left(2-M^{2}\right)\right) / 4 a_{1} a_{2}\right) t$ and $a_{2}>0$.

If $M=0$, then (55) turns out to be

$$
u_{7,1}^{1}(x, y, t)=\frac{\left(a_{3}+a_{4}\right) l \xi}{2 a_{2}} \pm \sqrt{\frac{6}{a_{2}}} \ln (\sin (\xi)),
$$

where $\xi=x+l y-\left(\left(4 l^{2} a_{2} a_{5}-l^{2} a_{3}^{2}-2 l^{2} a_{3} a_{4}-l^{2} a_{4}^{2}-8 a_{2}\right) /\right.$ $\left.4 a_{1} a_{2}\right) t$ and $a_{2}>0$.

If $M=1$, then (55) reduces to

$$
u_{7,1}^{1}(x, y, t)=\frac{\left(a_{3}+a_{4}\right) l \xi}{2 a_{2}} \mp 2 \sqrt{\frac{6}{a_{2}}} \arctan \left(\mathrm{e}^{\xi}\right),
$$

where $\quad \xi=x+l y-\left(\left(4 l^{2} a_{2} a_{5}-l^{2} a_{3}^{2}-2 l^{2} a_{3} a_{4}-l^{2}\right.\right.$. $\left.\left.a_{4}^{2}-4 a_{2}\right) / 4 a_{1} a_{2}\right) t$ and $a_{2}>0$.
Case 8. When $l_{0}=1, l_{2}=2 M^{2}-1, l_{4}=-M^{2}\left(1-M^{2}\right)$, we get

$$
u_{8,1}^{1}(x, y, t)=\frac{\left(a_{3}+a_{4}\right) l \xi}{2 a_{2}} \pm \sqrt{\frac{6}{a_{2}}} \ln \left(\frac{\operatorname{nd}(\xi)-\operatorname{cd}(\xi)}{\operatorname{sd}(\xi)}\right),
$$

where $\xi=x+l y-\left(\left(4 l^{2} a_{2} a_{5}-l^{2} a_{3}^{2}-2 l^{2} a_{3} a_{4}-l^{2} a_{4}^{2}-4 a_{2}\right.\right.$. $\left.\left.\left(2 M^{2}-1\right)\right) / 4 a_{1} a_{2}\right) t$ and $a_{2}>0$.

If $M=0$, then (58) becomes the solution (42) with $\xi=$ $x+l y-\left(\left(4 l^{2} a_{2} a_{5}-l^{2} a_{3}^{2}-2 l^{2} a_{3} a_{4}-l^{2} a_{4}^{2}+4 a_{2}\right) / 4 a_{1} a_{2}\right) t$.

If $M=1$, then (58) becomes the solution (57) with $\xi=$ $x+l y-\left(\left(4 l^{2} a_{2} a_{5}-l^{2} a_{3}^{2}-2 l^{2} a_{3} a_{4}-l^{2} a_{4}^{2}-4 a_{2}\right) / 4 a_{1} a_{2}\right) t$.

Case 9. When $l_{0}=1-M^{2}, l_{2}=2-M^{2}, l_{4}=1$, we have

$$
u_{9,1}^{1}(x, y, t)=\frac{\left(a_{3}+a_{4}\right) l \xi}{2 a_{2}} \pm \sqrt{\frac{6}{a_{2}}} \ln \left(\frac{\sqrt{1-M^{2}} \mathrm{~ns}(\xi)+\mathrm{ds}(\xi)}{\operatorname{cs}(\xi)}\right) \text {, }
$$

where $\xi=x+l y-\left(\left(4 l^{2} a_{2} a_{5}-l^{2} a_{3}^{2}-2 l^{2} a_{3} a_{4}-l^{2} a_{4}^{2}-4 a_{2}\right.\right.$. $\left.\left.\left(2-M^{2}\right)\right) / 4 a_{1} a_{2}\right) t$ and $a_{2}>0$.

If $M=0$, then (59) can be reduced into

$$
u_{9,1}^{1}(x, y, t)=\frac{\left(a_{3}+a_{4}\right) l \xi}{2 a_{2}} \mp \sqrt{\frac{6}{a_{2}}} \ln (\cos (\xi)) \text {, }
$$

where $\xi=x+l y-\left(\left(4 l^{2} a_{2} a_{5}-l^{2} a_{3}^{2}-2 l^{2} a_{3} a_{4}-l^{2} a_{4}^{2}-8 a_{2}\right) /\right.$ $\left.4 a_{1} a_{2}\right) t$ and $a_{2}>0$.

If $M=1$, then (59) becomes the solution (46) with $\xi=$ $x+l y-\left(\left(4 l^{2} a_{2} a_{5}-l^{2} a_{3}^{2}-2 l^{2} a_{3} a_{4}-l^{2} a_{4}^{2}-4 a_{2}\right) / 4 a_{1} a_{2}\right) t$.

Case 10. When $l_{0}=-M^{2}\left(1-M^{2}\right), l_{2}=2 M^{2}-1, l_{4}=1$, we have

$$
u_{10,1}^{1}(x, y, t)=\frac{\left(a_{3}+a_{4}\right) l \xi}{2 a_{2}} \mp \sqrt{-\frac{6}{a_{2}}}\left(\sqrt{\frac{M^{2}-1}{(M \operatorname{sn}(\xi))^{2}-1}} \operatorname{dn}(\xi)\right) \arcsin (\operatorname{Mcd}(\xi))
$$

where $\xi=x+l y-\left(\left(4 l^{2} a_{2} a_{5}-l^{2} a_{3}^{2}-2 l^{2} a_{3} a_{4}-l^{2} a_{4}^{2}-4 a_{2}\right.\right.$. $\left.\left.\left(2 M^{2}-1\right)\right) / 4 a_{1} a_{2}\right) t$ and $a_{2}<0$.

If $M=0$, then (61) becomes the solution (46) with $\xi=$ $x+l y-\left(\left(4 l^{2} a_{2} a_{5}-l^{2} a_{3}^{2}-2 l^{2} a_{3} a_{4}-l^{2} a_{4}^{2}+4 a_{2}\right) / 4 a_{1} a_{2}\right) t$.
If $M=1$, then (61) turns out to be the solution (46) with $\xi=$ $x+l y-\left(1 / 4 a_{1} a_{2}\right)\left(4 l^{2} a_{2} a_{5}-l^{2} a_{3}^{2}-2 l^{2} a_{3} a_{4}-l^{2} a_{4}^{2}-4 a_{2}\right) t$.

Case 11. When $l_{0}=\left(1-M^{2}\right) / 4, l_{2}=\left(1+M^{2}\right) / 2, l_{4}=$ $\left(1-M^{2}\right) / 4$, we have

$$
\begin{aligned}
& u_{11,1}^{1}(x, y, t)=\frac{\left(a_{3}+a_{4}\right) l \xi}{2 a_{2}} \mp \sqrt{\frac{3}{2 a_{2}}} \ln \left(\frac{\mathrm{dc}(\xi)+\sqrt{1-M^{2}} \mathrm{nc}(\xi)}{\mathrm{dc}(\xi)+\sqrt{1-M^{2}} \mathrm{sc}(\xi)}\right) \text {, } \\
& u_{11,2}^{1}(x, y, t)=\frac{\left(a_{3}+a_{4}\right) l \xi}{2 a_{2}} \pm \sqrt{\frac{3}{2 a_{2}} \ln \left(\frac{\mathrm{dc}(\xi)+\sqrt{1-M^{2}}}{\mathrm{nc}(\xi)}\right)}
\end{aligned}
$$


where $\xi=x+l y-\left(\left(4 l^{2} a_{2} a_{5}-l^{2} a_{3}^{2}-2 l^{2} a_{3} a_{4}-l^{2} a_{4}^{2}-2 a_{2}\right.\right.$. $\left.\left.\left(M^{2}+1\right)\right) / 4 a_{1} a_{2}\right) t$ and $a_{2}>0$.

If $M=0$, then (62) and (63) reduce to

$$
\begin{aligned}
& u_{11,1}^{1}(x, y, t)=\frac{\left(a_{3}+a_{4}\right) l \xi}{2 a_{2}} \pm \sqrt{\frac{3}{2 a_{2}}} \ln (\sin (\xi)+1), \\
& u_{11,2}^{1}(x, y, t)=\frac{\left(a_{3}+a_{4}\right) l \xi}{2 a_{2}} \mp \sqrt{\frac{3}{2 a_{2}}} \ln (\sin (\xi)-1),
\end{aligned}
$$

respectively, where $\quad \xi=x+l y-\left(\left(4 l^{2} a_{2} a_{5}-l^{2} a_{3}^{2}-2\right.\right.$. $\left.\left.l^{2} a_{3} a_{4}-l^{2} a_{4}^{2}-2 a_{2}\right) / 4 a_{1} a_{2}\right) t$ and $a_{2}>0$.

If $M=1$, then both (62) and (63) become the solution (46) with $\xi=x+l y-\left(1 / 4 a_{1} a_{2}\right)\left(4 l^{2} a_{2} a_{5}-l^{2} a_{3}^{2}-2 l^{2} a_{3} a_{4}-\right.$ $\left.l^{2} a_{4}^{2}-4 a_{2}\right) t$.

Case 12. When $l_{0}=-\left(1-M^{2}\right)^{2} / 4, l_{2}=\left(1+M^{2}\right) / 2$, $l_{4}=-1 / 4$, we have

$$
\begin{aligned}
& u_{12,1}^{1}(x, y, t)=\frac{\left(a_{3}+a_{4}\right) l \xi}{2 a_{2}} \pm \sqrt{-\frac{3}{2 a_{2}}}(\arctan (M \operatorname{sd}(\xi))-\operatorname{am}(\xi)), \\
& u_{12,2}^{1}(x, y, t)=\frac{\left(a_{3}+a_{4}\right) l \xi}{2 a_{2}} \pm \sqrt{-\frac{3}{2 a_{2}}}(\arctan (\operatorname{Msd}(\xi))+\operatorname{am}(\xi)),
\end{aligned}
$$

where $\xi=x+l y-\left(\left(4 l^{2} a_{2} a_{5}-l^{2} a_{3}^{2}-2 l^{2} a_{3} a_{4}-l^{2} a_{4}^{2}-2 a_{2}\right.\right.$. $\left.\left.\left(1+M^{2}\right)\right) / 4 a_{1} a_{2} t\right)$ and $a_{2}<0$.

If $M=0$, then (66) and (67) are reduced into only one solution as follows:

$$
u_{12,1}^{1}(x, y, t)=\left(\frac{\left(a_{3}+a_{4}\right) l}{2 a_{2}} \pm \sqrt{-\frac{3}{2 a_{2}}}\right) \xi
$$

where $\xi=x+l y-\left(\left(4 l^{2} a_{2} a_{5}-l^{2} a_{3}^{2}-2 l^{2} a_{3} a_{4}-l^{2} a_{4}^{2}-2 a_{2}\right) /\right.$ $\left.4 a_{1} a_{2}\right) t$ and $a_{2}<0$.

If $M=1$, then both (66) and (67) become the solution (46) with $\xi=x+l y-\left(1 / 4 a_{1} a_{2}\right)\left(4 l^{2} a_{2} a_{5}-l^{2} a_{3}^{2}-2 l^{2} a_{3} a_{4}-\right.$ $\left.l^{2} a_{4}^{2}-4 a_{2}\right) t$ and $a_{2}<0$.

Case 13. When $l_{0}=1 / 4, l_{2}=\left(1-2 M^{2}\right) / 2, l_{4}=1 / 4$, we have

$$
\begin{aligned}
& u_{13,1}^{1}(x, y, t)=\frac{\left(a_{3}+a_{4}\right) l \xi}{2 a_{2}} \pm \sqrt{\frac{3}{2 a_{2}}} \ln \left(\frac{(\operatorname{sn}(\xi))^{2}}{(\operatorname{dn}(\xi)+1)(\operatorname{dn}(\xi)+\operatorname{cn}(\xi))}\right) \\
& u_{13,2}^{1}(x, y, t)=\frac{\left(a_{3}+a_{4}\right) l \xi}{2 a_{2}} \pm \sqrt{\frac{3}{2 a_{2}}} \ln \left(\frac{\mathrm{dn}(\xi)+1}{\operatorname{dn}(\xi)+\operatorname{cn}(\xi)}\right)
\end{aligned}
$$

where $\xi=x+l y-\left(\left(4 l^{2} a_{2} a_{5}-l^{2} a_{3}^{2}-2 l^{2} a_{3} a_{4}-l^{2} a_{4}^{2}-2 a_{2}\right.\right.$. $\left.\left.\left(1-2 M^{2}\right)\right) / 4 a_{1} a_{2}\right) t$ and $a_{2}>0$.

If $M=0$, then (69) and (70) become

$$
\begin{aligned}
& u_{13,1}^{1}(x, y, t)=\frac{\left(a_{3}+a_{4}\right) l \xi}{2 a_{2}} \pm \sqrt{\frac{3}{2 a_{2}}} \ln (1-\cos (\xi)), \\
& u_{13,2}^{1}(x, y, t)=\frac{\left(a_{3}+a_{4}\right) l \xi}{2 a_{2}} \pm \sqrt{\frac{3}{2 a_{2}}} \ln (1+\cos (\xi)),
\end{aligned}
$$

respectively, where $\quad \xi=x+l y-\left(\left(4 l^{2} a_{2} a_{5}-l^{2} a_{3}^{2}-2\right.\right.$. $\left.\left.l^{2} a_{3} a_{4}-l^{2} a_{4}^{2}-2 a_{2}\right) / 4 a_{1} a_{2}\right) t$ and $a_{2}>0$.
If $M=1$, then (69) and (70) turn out to be

$$
\begin{aligned}
u_{13,1}^{1}(x, y, t)= & \frac{\left(a_{3}+a_{4}\right) l \xi}{2 a_{2}} \pm \sqrt{\frac{6}{a_{2}}}\left(\operatorname{arctanh}\left(\mathrm{e}^{2 \xi}\right)\right. \\
& -\ln (\sinh (\xi))), \\
u_{13,2}^{1}(x, y, t)= & \frac{\left(a_{3}+a_{4}\right) l \xi}{2 a_{2}} \pm \sqrt{\frac{6}{a_{2}}}\left(\operatorname{arctanh}\left(\mathrm{e}^{2 \xi}\right)\right. \\
& +\ln (\sinh (\xi))),
\end{aligned}
$$


respectively, where $\xi=x+l y-\left(\left(4 l^{2} a_{2} a_{5}-l^{2} a_{3}^{2}-2 l^{2} a_{3} a_{4}\right.\right.$ $\left.\left.-l^{2} a_{4}^{2}+2 a_{2}\right) / 4 a_{1} a_{2}\right) t$ and $a_{2}>0$.
Case 14. When $l_{0}=\left(1-M^{2}\right) / 4, l_{2}=\left(1+M^{2}\right) / 2, l_{4}=$ $\left(1-M^{2}\right) / 4$, we have

$$
\begin{aligned}
& u_{14,1}^{1}(x, y, t)=\frac{\left(a_{3}+a_{4}\right) l \xi}{2 a_{2}} \mp \sqrt{\frac{6}{a_{2}}} \operatorname{arctanh}\left(\frac{1}{\sqrt{1-M^{2}}}\left(\frac{M^{2} \operatorname{sn}(\xi)}{\operatorname{dn}(\xi)+1}-1\right)\right), \\
& u_{14,2}^{1}(x, y, t)=\frac{\left(a_{3}+a_{4}\right) l \xi}{2 a_{2}} \mp \sqrt{\frac{6}{a_{2}}} \operatorname{arctanh}\left(\frac{1}{\sqrt{1-M^{2}}}\left(\frac{M^{2} \operatorname{sn}(\xi)}{\operatorname{dn}(\xi)+1}+1\right)\right),
\end{aligned}
$$

where $\xi=x+l y-\left(\left(4 l^{2} a_{2} a_{5}-l^{2} a_{3}^{2}-2 l^{2} a_{3} a_{4}-l^{2} a_{4}^{2}-2 a_{2}\right.\right.$. $\left.\left.\left(1+M^{2}\right)\right) / 4 a_{1} a_{2}\right) t$ and $a_{2}>0$.

If $M=0$, then both (75) and (76) turn out to be

$$
u_{14,1}^{1}(x, y, t)=\frac{\left(a_{3}+a_{4}\right) l \xi}{2 a_{2}} \pm \sqrt{\frac{3}{2 a_{2}}} \ln (1-\sin (\xi)) \text {, }
$$

where $\xi=x+l y-\left(\left(4 l^{2} a_{2} a_{5}-l^{2} a_{3}^{2}-2 l^{2} a_{3} a_{4}-l^{2} a_{4}^{2}-2 a_{2}\right) /\right.$ $\left.4 a_{1} a_{2}\right) t$ and $a_{2}>0$.

If $M=1$, then both (75) and (76) become the solution (46) with $\xi=x+l y-\left(1 / 4 a_{1} a_{2}\right)\left(4 l^{2} a_{2} a_{5}-l^{2} a_{3}^{2}-\right.$ $\left.2 l^{2} a_{3} a_{4}-l^{2} a_{4}^{2}-4 a_{2}\right) t$.

Case 15. When $l_{0}=1 / 4, l_{2}=\left(1+M^{2}\right) / 2, l_{4}=\left(1-M^{2}\right)^{2} / 4$, we have

$$
\begin{aligned}
& u_{15,1}^{1}(x, y, t)=\frac{\left(a_{3}+a_{4}\right) l \xi}{2 a_{2}} \pm \sqrt{\frac{3}{2 a_{2}}} \ln \left(\frac{(\operatorname{sn}(\xi))^{2}}{(\operatorname{dn}(\xi)+1)(\operatorname{cn}(\xi)+1)}\right) \\
& u_{15,2}^{1}(x, y, t)=\frac{\left(a_{3}+a_{4}\right) l \xi}{2 a_{2}} \pm \sqrt{\frac{3}{2 a_{2}}} \ln \left(\frac{\operatorname{cn}(\xi)+1}{\operatorname{dn}(\xi)+1}\right),
\end{aligned}
$$

where $\xi=x+l y-\left(\left(4 l^{2} a_{2} a_{5}-l^{2} a_{3}^{2}-2 l^{2} a_{3} a_{4}-l^{2} a_{4}^{2}-2 a_{2}\right.\right.$. $\left.\left.\left(1+M^{2}\right)\right) / 4 a_{1} a_{2}\right) t$ and $a_{2}>0$.

If $M=0$, then both (78) and (79) become the solution (71) with $\xi=x+l y-\left(1 / 4 a_{1} a_{2}\right)\left(4 l^{2} a_{2} a_{5}-l^{2} a_{3}^{2}-2 l^{2} a_{3} a_{4}-\right.$ $\left.l^{2} a_{4}^{2}-2 a_{2}\right) t$.

If $M=1$, then (78) and (79) are reduced into the solution (57) with $\xi=x+l y-\left(1 / 4 a_{1} a_{2}\right)\left(4 l^{2} a_{2} a_{5}-l^{2} a_{3}^{2}-2 l^{2} a_{3} a_{4}-\right.$ $\left.l^{2} a_{4}^{2}-4 a_{2}\right) t$.

Result 5

$$
\begin{aligned}
\alpha_{0} & =\frac{\left(a_{3}+a_{4}\right) l}{2 a_{2}}, \\
\alpha_{1} & = \pm \sqrt{\frac{6 l_{4}}{a_{2}}}, \\
\alpha_{-1} & =0, \\
\lambda & =\frac{l^{2}\left(a_{3}+a_{4}\right)^{2}-4 a_{2}\left(l^{2} a_{5}-l_{2}\right)}{4 a_{1} a_{2}}, \\
\delta & =-\frac{l\left(a_{3}+a_{4}\right)\left(l^{2}\left(a_{3}+a_{4}\right)^{2}+12 l_{2} a_{2}\right)}{24 a_{2}^{2}},
\end{aligned}
$$

where $a_{1}, a_{2}, a_{3}, a_{4}, a_{5}, l_{0}, l_{2}, l_{4}$, and $l$ are arbitrary constants, provided that $a_{1} a_{2} \neq 0, l_{4} a_{2}>0$.

Substituting (80) into (37), we get the solution of (18) as

$$
z(\xi)=\alpha_{0}+\alpha_{1} \psi(\xi)
$$

where $\psi(\xi)$ has the forms as expressed in (15) with $\xi=x+l y+\lambda t$, where $\lambda$ is defined in (80). In consequence, we can proceed the same process as shown above to obtain the solutions of (2) which are omitted here due to the minimalism.

Result 6

Result 6.1

$$
\begin{aligned}
\alpha_{0} & =\frac{\left(a_{3}+a_{4}\right) l}{2} a_{2}, \\
\alpha_{1} & =\sqrt{\frac{6 l_{4}}{a_{2}}}, \\
\alpha_{-1} & = \pm \sqrt{\frac{6 l_{0}}{a 2}}, \\
\lambda & =\frac{\left(a_{3}+a_{4}\right)^{2} l^{2}-4 a_{2}\left(l^{2} a_{5}+\alpha_{-1} \alpha_{1}-l_{2}\right)}{4 a_{1} a_{2}}, \\
\delta & =\frac{\left(a_{3}+a_{4}\right) l\left(12 a_{2}\left(\alpha_{-1} \alpha_{1} a_{2}-l_{2}\right)-\left(a_{3}+a_{4}\right)^{2} l^{2}\right)}{24 a_{2}^{2}},
\end{aligned}
$$

where $a_{1}, a_{2}, a_{3}, a_{4}, a_{5}, l_{0}, l_{2}, l_{4}$, and $l$ are arbitrary constants, provided that $a_{1} a_{2} \neq 0, l_{0} a_{2}>0, l_{4} a_{2}>0$.Using the obtained results (82), we have the following cases for the exact solutions of (2).

Case 16. When $l_{0}=1, l_{2}=-\left(1+M^{2}\right)$ and $l_{4}=M^{2}$, we obtain 


$$
\begin{aligned}
u_{1,1}^{3}(x, y, t)= & \frac{\left(a_{3}+a_{4}\right) l \xi}{2 a_{2}}+\sqrt{\frac{6}{a_{2}}} \ln (\operatorname{dn}(\xi)-\operatorname{Mcn}(\xi)) \\
& \pm \sqrt{\frac{6}{a_{2}}} \ln \left(\frac{\operatorname{sn}(\xi)}{\operatorname{cn}(\xi)+\operatorname{dn}(\xi)}\right), \\
u_{1,2}^{3}(x, y, t)= & \frac{\left(a_{3}+a_{4}\right) l \xi}{2 a_{2}}+\sqrt{\frac{6}{a_{2}}} \ln (\operatorname{nd}(\xi)+\operatorname{Msd}(\xi)) \\
& \pm \sqrt{\frac{6}{a_{2}}} \ln \left(\frac{\operatorname{nd}(\xi)+\operatorname{sd}(\xi)}{\operatorname{cd}(\xi)}\right),
\end{aligned}
$$

where $\quad \xi=x+l y+\left(\left(\left(a_{3}+a_{4}\right)^{2} l^{2}-4 l^{2} a_{2} a_{5}-4 \alpha_{-1} \alpha_{1} a_{2}^{2}-\right.\right.$ $\left.\left.4\left(M^{2}+1\right) a_{2}\right) / 4 a_{1} a_{2}\right) t$ and $a_{2}>0$.

If $M=0$, then solutions (83) and (84) reduce to the solutions (42) and (43), respectively, where $\xi=x+l y+$ $\left(\left(\left(a_{3}+a_{4}\right)^{2} l^{2}-4 l^{2} a_{2} a_{5}-4 \alpha_{-1} \alpha_{1} a_{2}^{2}-4 a_{2}\right) / 4 a_{1} a_{2}\right) t$.

If $M=1$, then both (83) and (84) become

$$
u_{1,1}^{3}(x, y, t)=\left(\frac{\left(a_{3}+a_{4}\right) l}{2 a_{2}}+2 \sqrt{\frac{6}{a_{2}}}\right) \xi
$$

where $\quad \xi=x+l y+\left(\left(\left(a_{3}+a_{4}\right)^{2} l^{2}-4 l^{2} a_{2} a_{5}-4 \alpha_{-1} \alpha_{1} a_{2}^{2}-\right.\right.$ $\left.\left.8 a_{2}\right) / 4 a_{1} a_{2}\right) t$ and $a_{2}>0$.

Case 17. When $l_{0}=1-M^{2}, l_{2}=2 M^{2}-1$ and $l_{4}=-M^{2}$, we have

$$
\begin{aligned}
u_{2,1}^{3}(x, y, t)= & \frac{\left(a_{3}+a_{4}\right) l \xi}{2 a_{2}}+\sqrt{\frac{6}{a_{2}}} \operatorname{arctanh}(i M \operatorname{sd}(\xi)) \\
& \pm \sqrt{\frac{6}{a_{2}}} \ln \left(\frac{\sqrt{1-M^{2}} \operatorname{sn}(\xi)+\operatorname{dn}(\xi)}{\operatorname{cn}(\xi)}\right),
\end{aligned}
$$

where $\xi=x+l y+\left(\left(\left(a_{3}+a_{4}\right)^{2} l^{2}-4 l^{2} a_{2} a_{5}-4 \alpha_{-1} \alpha_{1} a_{2}^{2}+4\right.\right.$. $\left.\left.\left(2 M^{2}-1\right) a_{2}\right) / 4 a_{1} a_{2}\right) t$ and $a_{2}>0$.

If $M=0$, then (86) becomes the solution (43) with $\xi=x+l y+\left(\left(\left(a_{3}+a_{4}\right)^{2} l^{2}-4 l^{2} a_{2} a_{5}-4 \alpha_{-1} \alpha_{1} a_{2}^{2}-4 a_{2}\right) /\right.$ $\left.4 a_{1} a_{2}\right) t$.

If $M=1$, then (86) turns out to be

$$
u_{2,1}^{3}(x, y, t)=\frac{\left(a_{3}+a_{4}\right) l \xi}{2 a_{2}}+\sqrt{-\frac{6}{a_{2}}} \arctan (\sinh (\xi)),
$$

where $\quad \xi=x+l y+\left(\left(a_{3}+a_{4}\right)^{2} l^{2}-4 l^{2} a_{2} a_{5}-4 \alpha_{-1} \alpha_{1} a_{2}^{2}+\right.$ $\left.4 a_{2} / 4 a_{1} a_{2}\right) t$ and $a_{2}<0$.

Case 18. When $l_{0}=M^{2}-1, l_{2}=2-M^{2}, l_{4}=-1$, we have

$$
\begin{aligned}
u_{3,1}^{3}(x, y, t)= & \frac{\left(a_{3}+a_{4}\right) l \xi}{2 a_{2}}+\sqrt{-\frac{6}{a_{2}}} \operatorname{am}(\xi) \\
& \pm \sqrt{-\frac{6}{a_{2}}} \arctan \left(\frac{\sqrt{1-M^{2}} \operatorname{sn}(\xi)}{\operatorname{cn}(\xi)}\right),
\end{aligned}
$$

where $\quad \xi=x+l y+\left(\left(\left(a_{3}+a_{4}\right)^{2} l^{2}-4 l^{2} a_{2} a_{5}-4 \alpha_{-1} \alpha_{1} a_{2}^{2}\right.\right.$ $\left.\left.+4\left(2-M^{2}\right) a_{2}\right) / 4 a_{1} a_{2}\right) t$ and $a_{2}<0$.

If $M=0$, then (88) becomes

$$
u_{3,1}^{3}(x, y, t)=\left(\frac{\left(a_{3}+a_{4}\right) l}{2 a_{2}}+2 \sqrt{-\frac{6}{a_{2}}}\right) \xi,
$$

where $\quad \xi=x+l y+\left(\left(\left(a_{3}+a_{4}\right)^{2} l^{2}-4 l^{2} a_{2} a_{5}-4 \alpha_{-1} \alpha_{1} a_{2}^{2}+\right.\right.$ $\left.\left.8 a_{2}\right) / 4 a_{1} a_{2}\right) t$ and $a_{2}<0$.

If $M=1$, then (88) becomes the solution (87) with $\xi=x+l y+\left(\left(\left(a_{3}+a_{4}\right)^{2} l^{2}-4 l^{2} a_{2} a_{5}-4 \alpha_{-1} \alpha_{1} a_{2}^{2}+4 a_{2}\right) /\right.$ $\left.4 a_{1} a_{2}\right) t$.

Case 19. When $l_{0}=M^{2}, l_{2}=-\left(1+M^{2}\right), l_{4}=1$, we have

$$
\begin{aligned}
u_{4,1}^{3}(x, y, t)= & \frac{\left(a_{3}+a_{4}\right) l \xi}{2 a_{2}}+\sqrt{\frac{6}{a_{2}}} \ln (\mathrm{ds}(\xi)-\operatorname{cs}(\xi)) \\
& \pm \sqrt{\frac{6}{a_{2}}} \ln \left(\frac{\mathrm{ds}(\xi)-M \operatorname{cs}(\xi)}{\operatorname{ns}(\xi)}\right), \\
u_{4,2}^{3}(x, y, t)= & \frac{\left(a_{3}+a_{4}\right) l \xi}{2 a_{2}}+\sqrt{\frac{6}{a_{2}}} \ln (\operatorname{nc}(\xi)-\operatorname{sc}(\xi)) \\
& \pm \sqrt{\frac{6}{a_{2}}} \ln \left(\frac{\operatorname{nc}(\xi)-M \operatorname{sc}(\xi)}{\operatorname{dc}(\xi)}\right),
\end{aligned}
$$

where $\xi=x+l y+\left(\left(\left(a_{3}+a_{4}\right)^{2} l^{2}-4 l^{2} a_{2} a_{5}-4 \alpha_{-1} \alpha_{1} a_{2}^{2}-4\right.\right.$. $\left.\left.\left(1+M^{2}\right) a_{2}\right) / 4 a_{1} a_{2}\right) t$ and $a_{2}>0$.

If $M=0$, then the solutions (90) and (91) reduce to the solutions (42) and (43), respectively, where $\xi=x+l y+$ $\left(\left(\left(a_{3}+a_{4}\right)^{2} l^{2}-4 l^{2} a_{2} a_{5}-4 \alpha_{-1} \alpha_{1} a_{2}^{2}-4 a_{2}\right) / 4 a_{1} a_{2}\right) t$.

If $M=1$, then both (90) and (91) become

$$
u_{4,1}^{3}(x, y, t)=\frac{\left(a_{3}+a_{4}\right) l \xi}{2 a_{2}}+\sqrt{\frac{6}{a_{2}}} \ln (\sinh (\xi)) \pm \sqrt{\frac{6}{a_{2}}} \ln (\cosh (\xi)),
$$

where $\quad \xi=x+l y+\left(\left(\left(a_{3}+a_{4}\right)^{2} l^{2}-4 l^{2} a_{2} a_{5}-4 \alpha_{-1} \alpha_{1} a_{2}^{2}-\right.\right.$ $\left.\left.8 a_{2}\right) / 4 a_{1} a_{2}\right) t$ and $a_{2}>0$.

Case 20. When $l_{0}=-M^{2}, l_{2}=2 M^{2}-1, l_{4}=1-M^{2}$, we have

$$
\begin{aligned}
u_{5,1}^{3}(x, y, t)= & \frac{\left(a_{3}+a_{4}\right) l \xi}{2 a_{2}}+\sqrt{\frac{6}{a_{2}}} \ln \left(\sqrt{1-M^{2}} \operatorname{sc}(\xi)+\mathrm{dc}(\xi)\right) \\
& \pm \sqrt{\frac{6}{a_{2}}} \operatorname{arctanh}(i M \operatorname{sd}(\xi)),
\end{aligned}
$$

where $\xi=x+l y+\left(\left(\left(a_{3}+a_{4}\right)^{2} l^{2}-4 l^{2} a_{2} a_{5}-4 \alpha_{-1} \alpha_{1} a_{2}^{2}+4\right.\right.$. $\left.\left.\left(2 M^{2}-1\right) a_{2}\right) / 4 a_{1} a_{2}\right) t$ and $a_{2}>0$.

If $M=0$, then (93) becomes the solution (43) with $\xi=x+l y+\left(\left(\left(a_{3}+a_{4}\right)^{2} l^{2}-4 l^{2} a_{2} a_{5}-4 \alpha_{-1} \alpha_{1} a_{2}^{2}-4 a_{2}\right) /\right.$ $\left.4 a_{1} a_{2}\right) t$. 
If $M=1$, then (93) turns out to be

$$
u_{5,1}^{3}(x, y, t)=\frac{\left(a_{3}+a_{4}\right) l \xi}{2 a_{2}} \pm 2 \sqrt{-\frac{6}{a_{2}}} \arctan \left(\mathrm{e}^{\xi}\right)
$$

where $\quad \xi=x+l y+\left(\left(\left(a_{3}+a_{4}\right)^{2} l^{2}-4 l^{2} a_{2} a_{5}-4 \alpha_{-1} \alpha_{1} a_{2}^{2}+\right.\right.$ $\left.\left.4 a_{2}\right) / 4 a_{1} a_{2}\right) t$ and $a_{2}<0$.

Case 21. When $l_{0}=-1, l_{2}=2-M^{2}, l_{4}=M^{2}-1$, we have

$$
\begin{aligned}
u_{6,1}^{3}(x, y, t)= & \frac{\left(a_{3}+a_{4}\right) l \xi}{2 a_{2}}+\sqrt{-\frac{6}{a_{2}}} \arctan \left(\sqrt{1-M^{2}} \operatorname{sc}(\xi)\right) \\
& \pm \sqrt{-\frac{6}{a_{2}}} \operatorname{am}(\xi)
\end{aligned}
$$

where $\xi=x+l y+\left(\left(\left(a_{3}+a_{4}\right)^{2} l^{2}-4 l^{2} a_{2} a_{5}-4 \alpha_{-1} \alpha_{1} a_{2}^{2}+4\right.\right.$. $\left.\left.\left(2-M^{2}\right) a_{2}\right) / 4 a_{1} a_{2}\right) t$ and $a_{2}<0$.

If $M=0$, then (95) becomes the solution (89) with $\xi=$ $x+l y+\left(\left(\left(a_{3}+a_{4}\right)^{2} l^{2}-4 l^{2} a_{2} a_{5}-4 \alpha_{-1} \alpha_{1} a_{2}^{2}+8 a_{2}\right) / 4 a_{1} a_{2}\right) t$.

If $M=1$, then (95) becomes the solution (94) with $\xi=$ $x+l y+\left(\left(\left(a_{3}+a_{4}\right)^{2} l^{2}-4 l^{2} a_{2} a_{5}-4 \alpha_{-1} \alpha_{1} a_{2}^{2}+4 a_{2}\right) / 4 a_{1} a_{2}\right) t$.

Case 22. When $l_{0}=1, l_{2}=2-M^{2}, l_{4}=1-M^{2}$, we obtain

$$
\begin{aligned}
u_{7,1}^{3}(x, y, t)= & \frac{\left(a_{3}+a_{4}\right) l \xi}{2 a_{2}}+\sqrt{\frac{6}{a_{2}}} \ln \left(\mathrm{dc}(\xi)+\sqrt{1-M^{2}} \mathrm{nc}(\xi)\right) \\
& \pm \sqrt{\frac{6}{a_{2}}} \ln \left(\frac{\operatorname{nc}(\xi)-\mathrm{dc}(\xi)}{\operatorname{sc}(\xi)}\right),
\end{aligned}
$$

where $\xi=x+l y+\left(\left(\left(a_{3}+a_{4}\right)^{2} l^{2}-4 l^{2} a_{2} a_{5}-4 \alpha_{-1} \alpha_{1} a_{2}^{2}+4\right.\right.$. $\left.\left.\left(2-M^{2}\right) a_{2}\right) / 4 a_{1} a_{2}\right) t$ and $a_{2}>0$.

If $M=0$, then (96) reduces to

$$
\begin{aligned}
u_{7,1}^{3}(x, y, t)= & \frac{\left(a_{3}+a_{4}\right) l \xi}{2 a_{2}}-\sqrt{\frac{6}{a_{2}}} \ln (\cos (\xi)) \\
& \pm \sqrt{\frac{6}{a_{2}}} \ln (\sin (\xi)),
\end{aligned}
$$

where $\quad \xi=x+l y+\left(\left(\left(a_{3}+a_{4}\right)^{2} l^{2}-4 l^{2} a_{2} a_{5}-4 \alpha_{-1} \alpha_{1} a_{2}^{2}+\right.\right.$ $\left.\left.8 a_{2}\right) / 4 a_{1} a_{2}\right) t$ and $a_{2}>0$.

If $M=1$, then (97) turns out to be

$$
u_{7,1}^{3}(x, y, t)=\frac{\left(a_{3}+a_{4}\right) l \xi}{2 a_{2}} \mp 2 \sqrt{\frac{6}{a_{2}}} \operatorname{arctanh}\left(\mathrm{e}^{\xi}\right),
$$

where $\quad \xi=x+l y+\left(\left(\left(a_{3}+a_{4}\right)^{2} l^{2}-4 l^{2} a_{2} a_{5}-4 \alpha_{-1} \alpha_{1} a_{2}^{2}+\right.\right.$ $\left.\left.4 a_{2}\right) / 4 a_{1} a_{2}\right) t$ and $a_{2}>0$.

Case 23. When $l_{0}=1, l_{2}=2 M^{2}-1, l_{4}=-M^{2}\left(1-M^{2}\right)$, we have

$$
u_{8,1}^{3}(x, y, t)=\frac{\left(a_{3}+a_{4}\right) l \xi}{2 a_{2}}-\sqrt{\frac{6}{a_{2}}}\left(\frac{\arcsin (\operatorname{Mcd}(\xi)) \sqrt{(M c d(\xi))^{2}-1}}{\operatorname{nd}(\xi)}\right) \pm \sqrt{\frac{6}{a_{2}}} \ln \left(\frac{\operatorname{nd}(\xi)-\operatorname{cd}(\xi)}{\operatorname{sd}(\xi)}\right)
$$

where $\xi=x+l y+\left(\left(\left(a_{3}+a_{4}\right)^{2} l^{2}-4 l^{2} a_{2} a_{5}-4 \alpha_{-1} \alpha_{1} a_{2}^{2}+4\right.\right.$. $\left.\left.\left(M^{2}-1\right) a_{2}\right) / 4 a_{1} a_{2}\right) t$ and $a_{2}>0$.

If $M=0$, then (99) becomes the solution (42), where $\xi=$ $x+l y+\left(1 / 4 a_{1} a_{2}\right)\left(\left(a_{3}+a_{4}\right)^{2} l^{2}-4 l^{2} a_{2} a_{5}-4 \alpha_{-1} \alpha_{1} a_{2}^{2}-4 a_{2}\right) t$.

If $M=1$, then (99) reduces to the solution (98) with $\xi=x+l y+\left(1 / 4 a_{1} a_{2}\right)\left(\left(a_{3}+a_{4}\right)^{2} l^{2}-4 l^{2} a_{2} a_{5}-4 \alpha_{-1} \alpha_{1} a_{2}^{2}\right) t$.

Case 24. When $l_{0}=1-M^{2}, l_{2}=2-M^{2}, l_{4}=1$, we have

$$
\begin{aligned}
u_{9,1}^{3}(x, y, t)= & \frac{\left(a_{3}+a_{4}\right) l \xi}{2 a_{2}}+\sqrt{\frac{6}{a_{2}}} \ln (\mathrm{ns}(\xi)-\mathrm{ds}(\xi)) \\
& \pm \sqrt{\frac{6}{a_{2}}} \ln \left(\mathrm{dc}(\xi)+\frac{\sqrt{1-M^{2}} \mathrm{~ns}(\xi)}{\operatorname{cs}(\xi)}\right),
\end{aligned}
$$

where $\xi=x+l y+\left(\left(\left(a_{3}+a_{4}\right)^{2} l^{2}-4 l^{2} a_{2} a_{5}-4 \alpha_{-1} \alpha_{1} a_{2}^{2}+4\right.\right.$. $\left.\left.\left(2-M^{2}\right) a_{2}\right) / 4 a_{1} a_{2}\right) t$ and $a_{2}>0$.

If $M=0$, then (100) becomes

$$
u_{9,1}^{3}(x, y, t)=\frac{\left(a_{3}+a_{4}\right) l \xi}{2 a_{2}}-\sqrt{\frac{6}{a_{2}}} \ln (\cos (\xi)) \pm \sqrt{\frac{6}{a_{2}}} \ln (\sin (\xi)),
$$

where $\quad \xi=x+l y+\left(\left(\left(a_{3}+a_{4}\right)^{2} l^{2}-4 l^{2} a_{2} a_{5}-4 \alpha_{-1} \alpha_{1} a_{2}^{2}+\right.\right.$ $\left.\left.8 a_{2}\right) / 4 a_{1} a_{2}\right) t$ and $a_{2}>0$.

If $M=1$, then (100) turns out to be

$$
u_{9,1}^{3}(x, y, t)=\frac{\left(a_{3}+a_{4}\right) l \xi}{2 a_{2}}+\sqrt{\frac{6}{a_{2}}} \ln \left(\tanh \left(\frac{\xi}{2}\right)\right) \text {, }
$$

where $\quad \xi=x+l y+\left(\left(\left(a_{3}+a_{4}\right)^{2} l^{2}-4 l^{2} a_{2} a_{5}-4 \alpha_{-1} \alpha_{1} a_{2}^{2}+\right.\right.$ $\left.\left.4 a_{2}\right) / 4 a_{1} a_{2}\right) t$ and $a_{2}>0$. 
Case 25. When $l_{0}=-M^{2}\left(1-M^{2}\right), l_{2}=2 M^{2}-1, l_{4}=1$, we get

$$
\begin{aligned}
u_{10,1}^{3}(x, y, t)= & \frac{\left(a_{3}+a_{4}\right) l \xi}{2 a_{2}}+\sqrt{\frac{6}{a_{2}}} \ln (\mathrm{ns}(\xi)-\operatorname{cs}(\xi)) \\
& \mp \sqrt{\frac{6}{a_{2}}}\left(\sqrt{\frac{M^{2}-1}{(M \operatorname{sn}(\xi))^{2}-1}} i \operatorname{dn}(\xi)\right) \arcsin (M \operatorname{cd}(\xi)),
\end{aligned}
$$

where $\xi=x+l y+\left(\left(\left(a_{3}+a_{4}\right)^{2} l^{2}-4 l^{2} a_{2} a_{5}-4 \alpha_{-1} \alpha_{1} a_{2}^{2}+4\right.\right.$. $\left.\left.\left(2 M^{2}-1\right) a_{2}\right) / 4 a_{1} a_{2}\right) t$ and $a_{2}>0$.

If $M=0$, then (103) reduces to

$$
u_{10,1}^{3}(x, y, t)=\frac{\left(a_{3}+a_{4}\right) l \xi}{2 a_{2}}-\sqrt{\frac{6}{a_{2}}} \ln (\csc (\xi)+\cot (\xi)) \text {, }
$$

where $\quad \xi=x+l y+\left(\left(\left(a_{3}+a_{4}\right)^{2} l^{2}-4 l^{2} a_{2} a_{5}-4 \alpha_{-1} \alpha_{1} a_{2}^{2}-\right.\right.$ $\left.\left.4 a_{2}\right) / 4 a_{1} a_{2}\right) t$ and $a_{2}>0$.

If $M=1$, then (103) becomes the solution (102) with $\xi=$ $x+l y+\left(\left(\left(a_{3}+a_{4}\right)^{2} l^{2}-4 l^{2} a_{2} a_{5}-4 \alpha_{-1} \alpha_{1} a_{2}^{2}+4 a_{2}\right) / 4 a_{1} a_{2}\right) t$.

Case 26. When $l_{0}=\left(1-M^{2}\right) / 4, l_{2}=\left(1+M^{2}\right) / 2, l_{4}=$ $\left(1-M^{2}\right) / 4$, we have

$$
\begin{aligned}
& u_{11,1}^{3}(x, y, t)=\frac{\left(a_{3}+a_{4}\right) l \xi}{2 a_{2}}+\sqrt{\frac{6}{a_{2}}} \ln \left(\sqrt{1-M^{2}} \operatorname{sc}(\xi)+\mathrm{dc}(\xi)\right), \\
& u_{11,2}^{3}(x, y, t)=\frac{\left(a_{3}+a_{4}\right) l \xi}{2 a_{2}} \pm \sqrt{\frac{6}{a_{2}}} \ln \left(\sqrt{1-M^{2}} \operatorname{nc}(\xi)+\operatorname{dc}(\xi)\right),
\end{aligned}
$$

where $\xi=x+l y+\left(\left(\left(a_{3}+a_{4}\right)^{2} l^{2}-4 l^{2} a_{2} a_{5}-4 \alpha_{-1} \alpha_{1} a_{2}^{2}+2\right.\right.$. $\left.\left.\left(M^{2}+1\right) a_{2}\right) / 4 a_{1} a_{2}\right) t$ and $a_{2}>0$.

If $M=0$, then (105) and (106) turn out to be

$$
\begin{aligned}
u_{11,1}^{3}(x, y, t)= & \frac{\left(a_{3}+a_{4}\right) l \xi}{2 a_{2}}-\sqrt{\frac{3}{2 a_{2}}} \ln (1-\sin (\xi)) \\
& \pm \sqrt{\frac{3}{2 a_{2}}} \ln (1+\sin (\xi)), \\
u_{11,2}^{3}(x, y, t)= & \frac{\left(a_{3}+a_{4}\right) l \xi}{2 a_{2}}-\sqrt{\frac{3}{2 a_{2}}} \ln (\sin (\xi)+1) \\
& \pm \sqrt{\frac{3}{2 a_{2}}} \ln (\sin (\xi)-1),
\end{aligned}
$$

respectively, where $\xi=x+l y+\left(\left(\left(a_{3}+a_{4}\right)^{2} l^{2}-4 l^{2} a_{2} a_{5}-\right.\right.$ $\left.\left.4 \alpha_{-1} \alpha_{1} a_{2}^{2}+2 a_{2}\right) / 4 a_{1} a_{2}\right) t$ and $a_{2}>0$.

If $M=1$, then both (105) and (106) become the solution (46) with $\xi=x+l y+\left(1 / 4 a_{1} a_{2}\right)\left(\left(a_{3}+a_{4}\right)^{2} l^{2}-4 l^{2} a_{2} a_{5}-\right.$ $\left.4 \alpha_{-1} \alpha_{1} a_{2}^{2}+4 a_{2}\right) t$.

Case 27. When $l_{0}=-\left(1-M^{2}\right)^{2} / 4, l_{2}=\left(1+M^{2}\right) / 2, l_{4}=$ $-1 / 4$, we have

$$
\begin{aligned}
& u_{12,1}^{3}(x, y, t)=\frac{\left(a_{3}+a_{4}\right) l \xi}{2 a_{2}}+\sqrt{\frac{6}{a_{2}}} \arctan \left(\frac{M \sin (\xi)}{\operatorname{dn}(\xi)}\right), \\
& u_{12,2}^{3}(x, y, t)=\frac{\left(a_{3}+a_{4}\right) l \xi}{2 a_{2}} \pm \sqrt{\frac{6}{a_{2}}} \operatorname{am}(\xi),
\end{aligned}
$$

where $\xi=x+l y+\left(\left(\left(a_{3}+a_{4}\right)^{2} l^{2}-4 l^{2} a_{2} a_{5}-4 \alpha_{-1} \alpha_{1} a_{2}^{2}+2\right.\right.$. $\left.\left.\left(M^{2}+1\right) a_{2}\right) / 4 a_{1} a_{2}\right) t$ and $a_{2}>0$.

If $M=0$, then the solutions (109) and (110) reduce to the solutions (46) and (48), respectively, where $\xi=x+l y+$ $\left(\left(\left(a_{3}+a_{4}\right)^{2} l^{2}-4 l^{2} a_{2} a_{5}-4 \alpha_{-1} \alpha_{1} a_{2}^{2}+2 a_{2}\right) / 4 a_{1} a_{2}\right) t$.

If $M=1$, then both (109) and (110) become the solution (87) with $\xi=x+l y+\left(1 / 4 a_{1} a_{2}\right)\left(\left(a_{3}+a_{4}\right)^{2} l^{2}-4 l^{2} a_{2} a_{5}-\right.$ $\left.4 \alpha_{-1} \alpha_{1} a_{2}^{2}+4 a_{2}\right) t$.

Case 28. When $l_{0}=1 / 4, l_{2}=\left(1-2 M^{2}\right) / 2, l_{4}=1 / 4$, we have

$$
u_{13,1}^{3}(x, y, t)=\frac{\left(a_{3}+a_{4}\right) l \xi}{2 a_{2}} \pm \sqrt{\frac{6}{a_{2}}} \ln \left(\frac{\operatorname{sn}(\xi)}{\operatorname{cn}(\xi)+\operatorname{dn}(\xi)}\right)
$$

$$
u_{13,2}^{3}(x, y, t)=\frac{\left(a_{3}+a_{4}\right) l \xi}{2 a_{2}}+\sqrt{\frac{6}{a_{2}}} \ln \left(\frac{\operatorname{sn}(\xi)}{\operatorname{dn}(\xi)+1}\right)
$$

where $\quad \xi=x+l y+\left(\left(\left(a_{3}+a_{4}\right)^{2} l^{2}-4 l^{2} a_{2} a_{5}-4 \alpha_{-1} \alpha_{1} a_{2}^{2}+\right.\right.$ $\left.\left.2\left(1-2 M^{2}\right) a_{2}\right) / 4 a_{1} a_{2}\right) t$ and $a_{2}>0$.

If $M=0$, then both (111) and (112) reduce to

$$
\begin{aligned}
u_{13,1}^{3}(x, y, t)= & \frac{\left(a_{3}+a_{4}\right) l \xi}{2 a_{2}}-\sqrt{\frac{3}{2 a_{2}}} \ln (1+\cos (\xi)) \\
& \pm \sqrt{\frac{3}{2 a_{2}}} \ln (1-\cos (\xi))
\end{aligned}
$$

where $\quad \xi=x+l y+\left(\left(\left(a_{3}+a_{4}\right)^{2} l^{2}-4 l^{2} a_{2} a_{5}-4 \alpha_{-1} \alpha_{1} a_{2}^{2}+\right.\right.$ $\left.\left.2 a_{2}\right) / 4 a_{1} a_{2}\right) t$ and $a_{2}>0$.

If $M=1$, then (111) and (112) become

$$
\begin{aligned}
u_{13,1}^{3}(x, y, t)= & \frac{\left(a_{3}+a_{4}\right) l \xi}{2 a_{2}}+\sqrt{\frac{3}{2 a_{2}}} \ln (1+\cosh (\xi)) \\
& \pm\left(\sqrt{\frac{3}{2 a_{2}}} \ln (\sinh (\xi))-\sqrt{\frac{6}{a_{2}}} \operatorname{arctanh}\left(\mathrm{e}^{\xi}\right)\right)
\end{aligned}
$$

$$
\begin{aligned}
u_{13,2}^{3}(x, y, t)= & \frac{\left(a_{3}+a_{4}\right) l \xi}{2 a_{2}}+\sqrt{\frac{3}{2 a_{2}}} \ln (1-\cosh (\xi)) \\
& \pm\left(\sqrt{\frac{3}{2 a_{2}}} \ln (\sinh (\xi))-\sqrt{\frac{6}{a_{2}}} \operatorname{arctanh}\left(\mathrm{e}^{\xi}\right)\right),
\end{aligned}
$$


respectively, where $\xi=x+l y+\left(\left(\left(a_{3}+a_{4}\right)^{2} l^{2}-4 l^{2} a_{2} a_{5}-\right.\right.$ $\left.\left.4 \alpha_{-1} \alpha_{1} a_{2}^{2}-2 a_{2}\right) / 4 a_{1} a_{2}\right) t$ and $a_{2}>0$.

Case 29. When $l_{0}=\left(1-M^{2}\right) / 4, l_{2}=\left(1+M^{2}\right) / 2, l_{4}=$ $\left(1-M^{2}\right) / 4$, we have

$$
\begin{aligned}
u_{14,1}^{3}(x, y, t)= & \frac{\left(a_{3}+a_{4}\right) l \xi}{2 a_{2}}+\sqrt{\frac{6}{a_{2}}} \operatorname{arctanh}\left(\frac{1}{\sqrt{1-M^{2}}}\right. \\
& \left.\cdot\left(\frac{M^{2} \operatorname{sn}(\xi)}{\operatorname{dn}(\xi)+1}+1\right)\right) \pm \sqrt{\frac{6}{a_{2}}} \operatorname{arctanh} \\
& \cdot\left(\frac{1}{\sqrt{1-M^{2}}}\left(\frac{M^{2} \operatorname{sn}(\xi)}{\operatorname{dn}(\xi)+1}-1\right)\right),
\end{aligned}
$$

where $\xi=x+l y+\left(\left(\left(a_{3}+a_{4}\right)^{2} l^{2}-4 l^{2} a_{2} a_{5}-4 \alpha_{-1} \alpha_{1} a_{2}^{2}+2\right.\right.$. $\left.\left.\left(1+M^{2}\right) a_{2}\right) / 4 a_{1} a_{2}\right) t$ and $a_{2}>0$.

If $M=0$, then (116) turns out to be

$$
\begin{aligned}
u_{14,1}^{3}(x, y, t)= & \frac{\left(a_{3}+a_{4}\right) l \xi}{2 a_{2}}+\sqrt{\frac{3}{2 a_{2}}} \ln (1+\sin (\xi)) \\
& \mp \sqrt{\frac{3}{2 a_{2}}} \ln (1-\sin (\xi)),
\end{aligned}
$$

where $\quad \xi=x+l y+\left(\left(\left(a_{3}+a_{4}\right)^{2} l^{2}-4 l^{2} a_{2} a_{5}-4 \alpha_{-1} \alpha_{1} a_{2}^{2}+\right.\right.$ $\left.\left.2 a_{2}\right) / 4 a_{1} a_{2}\right) t$ and $a_{2}>0$.

If $M=1$, then (116) is the same as the solution (46) with $\xi=x+l y+\left(1 / 4 a_{1} a_{2}\right)\left(\left(a_{3}+a_{4}\right)^{2} l^{2}-4 l^{2} a_{2} a_{5}-4 \alpha_{-1} \alpha_{1} a_{2}^{2}+\right.$ $\left.4 a_{2}\right) t$.

Case 30. When $l_{0}=1 / 4, l_{2}=\left(1+M^{2}\right) / 2, l_{4}=\left(1-M^{2}\right)^{2} / 4$, we have

$$
\begin{aligned}
& u_{15,1}^{3}(x, y, t)=\frac{\left(a_{3}+a_{4}\right) l \xi}{2 a_{2}}+\sqrt{\frac{6}{a_{2}}} \ln \left(\frac{\operatorname{sn}(\xi)}{\operatorname{dn}(\xi)+1}\right), \\
& u_{15,2}^{3}(x, y, t)=\frac{\left(a_{3}+a_{4}\right) l \xi}{2 a_{2}}+\sqrt{\frac{6}{a_{2}}} \ln \left(\frac{\operatorname{sn}(\xi)}{\operatorname{cn}(\xi)+1}\right),
\end{aligned}
$$

where $\xi=x+l y+\left(\left(\left(a_{3}+a_{4}\right)^{2} l^{2}-4 l^{2} a_{2} a_{5}-4 \alpha_{-1} \alpha_{1} a_{2}^{2}+2\right.\right.$. $\left.\left.\left(1+M^{2}\right) a_{2}\right) / 4 a_{1} a_{2}\right) t$ and $a_{2}>0$.

If $M=0$, then both (118) and (119) become

$$
\begin{aligned}
u_{15,1}^{3}(x, y, t)= & \frac{\left(a_{3}+a_{4}\right) l \xi}{2 a_{2}}+\sqrt{\frac{3}{2 a_{2}}} \ln (\cos (\xi)-1) \\
& \mp \sqrt{\frac{3}{2 a_{2}}} \ln (\cos (\xi)+1),
\end{aligned}
$$

where $\quad \xi=x+l y+\left(\left(\left(a_{3}+a_{4}\right)^{2} l^{2}-4 l^{2} a_{2} a_{5}-4 \alpha_{-1} \alpha_{1} a_{2}^{2}+\right.\right.$ $\left.\left.2 a_{2}\right) / 4 a_{1} a_{2}\right) t$ and $a_{2}>0$.

If $M=1$, then both (118) and (119) are the same as the solution (98) with $\xi=x+l y+\left(\left(\left(a_{3}+a_{4}\right)^{2} l^{2}-4 l^{2}\right.\right.$. $\left.\left.a_{2} a_{5}-4 \alpha_{-1} \alpha_{1} a_{2}^{2}+4 a_{2}\right) / 4 a_{1} a_{2}\right) t$.
Result 6.2.

$$
\begin{aligned}
\alpha_{0} & =\frac{\left(a_{3}+a_{4}\right) l}{2 a_{2}}, \\
\alpha_{1} & =-\sqrt{\frac{6 l_{4}}{a_{2}}}, \\
\alpha_{-1} & = \pm \sqrt{\frac{6 l_{0}}{a_{2}}}, \\
\lambda & =\frac{\left(a_{3}+a_{4}\right)^{2} l^{2}-4 a_{2}\left(l^{2} a_{5}+\alpha_{-1} \alpha_{1}-l_{2}\right)}{4 a_{1} a_{2}}, \\
\delta & =\frac{\left(a_{3}+a_{4}\right) l\left(12 a_{2}\left(\alpha_{-1} \alpha_{1} a_{2}-l_{2}\right)-\left(a_{3}+a_{4}\right)^{2} l^{2}\right)}{24 a_{2}^{2}},
\end{aligned}
$$

where $a_{1}, a_{2}, a_{3}, a_{4}, a_{5}, l_{0}, l_{2}, l_{4}$, and $l$ are arbitrary constants, provided that $a_{1} a_{2} \neq 0, l_{0} a_{2}>0, l_{4} a_{2}>0$.Substituting (121) into (37), then the solution of (18) becomes

$$
z(\xi)=\alpha_{0}+\alpha_{1} \psi(\xi)+\alpha_{-1} \psi(\xi)^{-1},
$$

where $\psi(\xi)$ has the forms expressed in (15) with $\xi=x-l y+\lambda t$, where $\lambda$ is defined in (121). Consequently, we can achieve many similar solutions of (2) using the same procedure as shown above. However, they are ignored here due to the minimalism.

\section{Some Graphical Representations of the Obtained Exact Solutions}

In this section, we will show graphical representations of the selected exact solutions, which have been constructed using the $\left(G^{\prime} / G^{2}\right)$-expansion method and the Jacobi elliptic equation method, for the $(2+1)$-dimensional Jaulent-Miodek equation (2). In addition, we will also discuss their physical explanations. The domain for plotting the chosen exact solutions of the equation is $-10 \leq x \leq 10$ and $-10 \leq t \leq 10$, but $y=0$ is fixed.

For the $\left(G^{\prime} / G^{2}\right)$-expansion method, only two solutions (23) and (26) are selected in order to show their graphical behaviors using $a_{1}=1, a_{2}=6, a_{3}=1, a_{4}=1, a_{5}=-1, l=$ $1, A=2, B=1, \mu=2$, and $\omega=2$ on the above domain. The graph of the exact solution $u_{1}^{1}(x, y, t)$ in (23) is depicted in Figure 1(a). It represents the singular periodic traveling wave solution. Similarly, the exact solution $u_{3}^{1}(x, y, t)$ in (26) is plotted in Figure 1(b) using the different set of the parameter values: $a_{1}=1, a_{2}=6, a_{3}=1, a_{4}=1, a_{5}=-1, l=1, A=2$, $B=1$, and $\omega=-1$. The graph demonstrates discontinuities of solution (26) on the domain.

For the Jacobi elliptic equation method, exact solutions (51), (52), and (116) of (2) are plotted using the distinct values of the modulus $M$ but utilizing the same set of the parameter values: $a_{1}=1, a_{2}=6, a_{3}=1, a_{4}=5, a_{5}=1$, $l=1$. Solution (51) with $M=1$ is portrayed in Figure 2(a), in which the symmetrical peakons is characterized. The graph 


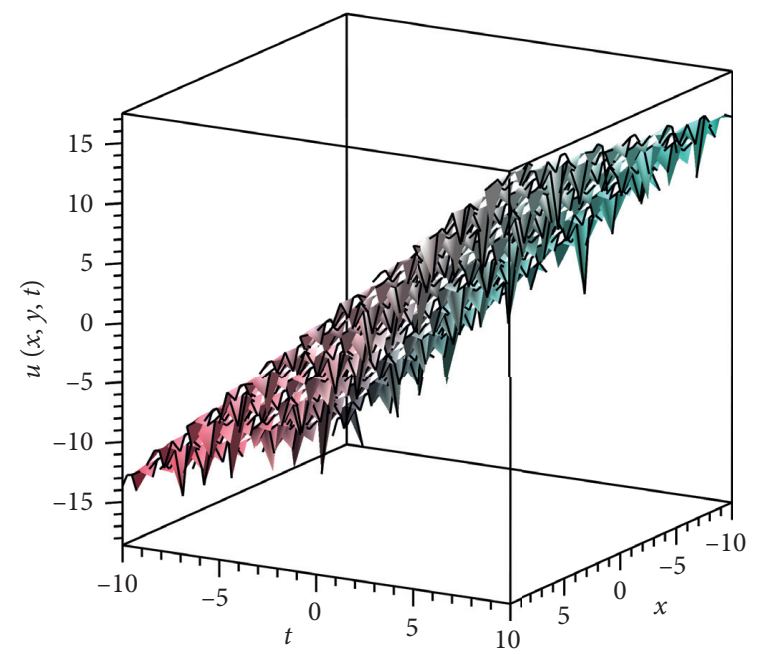

(a)

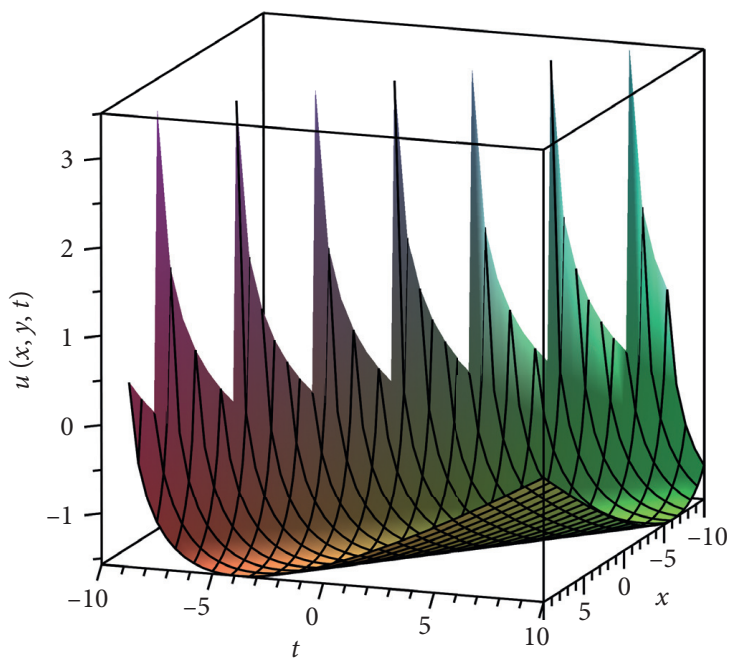

(b)

Figure 1: Plots of the solutions of (2) obtained using the $\left(G^{\prime} / G^{2}\right)$-expansion method and setting $y=0$ : (a) solution (23) and (b) solution (29).

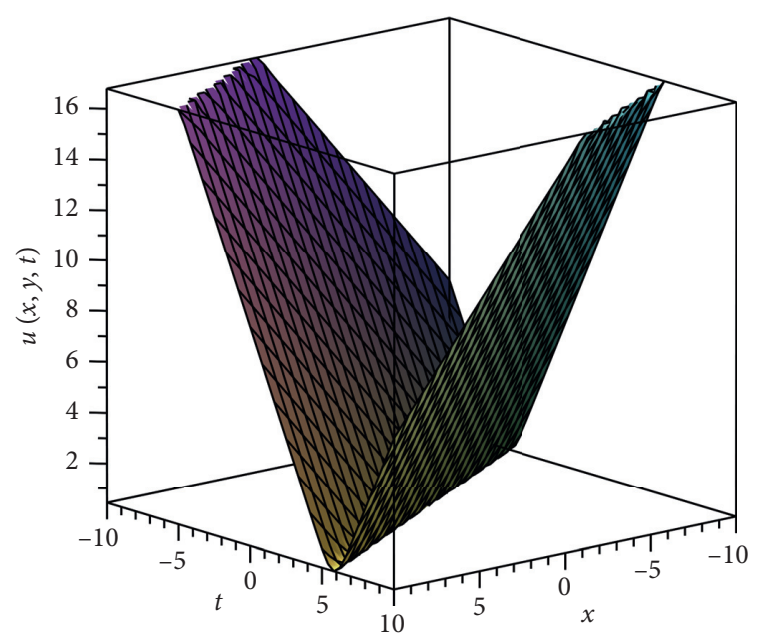

(a)

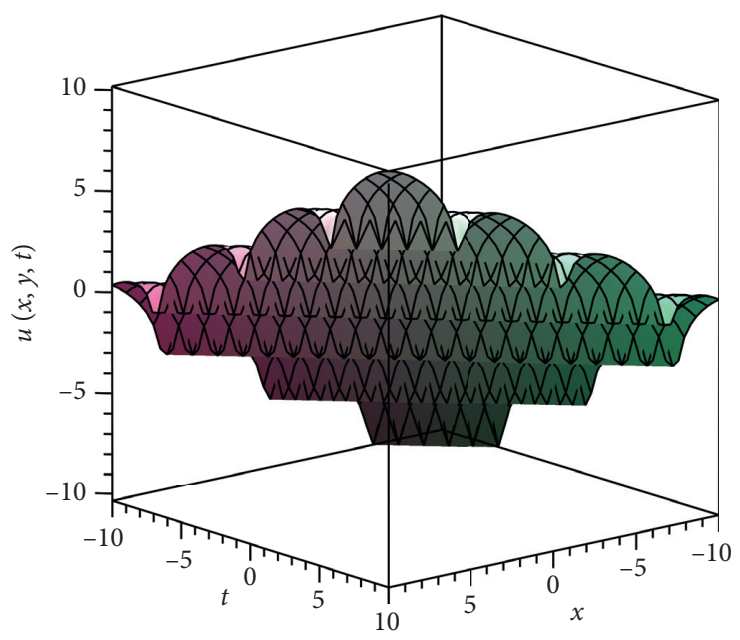

(b)

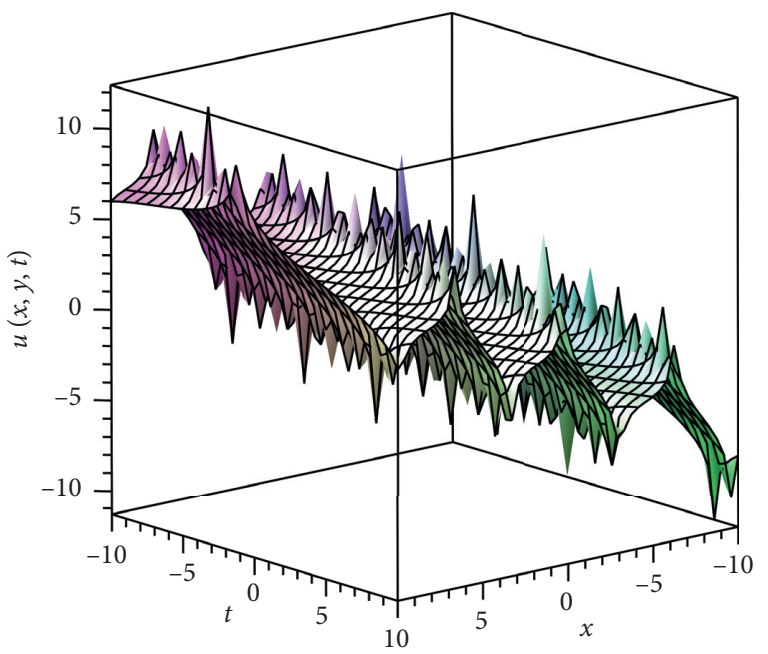

(c)

Figure 2: Plots of the solutions of (2) obtained using the Jacobi elliptic equation method and setting $y=0$ : (a) solution (51) with $M=1$, (b) solution (64) with $M=0$, and (c) solution (116) with $M=1 / 3$. 
of the exact solution (52) with $M=0$ is shown in Figure 2(b) describing the periodic traveling wave solution. In addition, Figure 2(c) shows the graphical result of the Jacobi elliptic function solution (116) with $M=1 / 3$. This graph gives singularities on the domain.

\section{Discussions and Conclusions}

In this article, we have utilized the two methods, namely, the $\left(G^{\prime} / G^{2}\right)$-expansion method and the Jacobi elliptic equation method to compute the explicit exact traveling wave solutions of the $(2+1)$-dimensional Jaulent-Miodek equation as given by (2). Applying the $\left(G^{\prime} / G^{2}\right)$-expansion method to the equation, we have obtained the different three sets of the parameter values from which the exact solutions have been formed. The obtained solutions include the trigonometric, hyperbolic, and rational function solutions. Employing the Jacobi elliptic equation method to analytically solve (2), three sets of the parameter values have been attained. Each set has consequently generated fifteen exact solutions in terms of the Jacobi elliptic function solutions depending upon the modulus $M \in[0,1]$. In particular, the trigonometric and hyperbolic function solutions of (2) can be reduced from the Jacobi elliptic function solutions when $M$ is replaced by 0 and 1, respectively. In Section 4, we have given some figures describing the behaviors of the chosen solutions of (2), e.g., the singular periodic traveling wave solution. All of the exact solutions, constructed by the two methods, have been achieved and verified by putting them back into the original problems with the help of the Maple 17 package program. The following results of (2) are brought from the previous literature to compare with our solutions.

(a) In [42], the authors used the direct symmetry method to obtain the exact solutions of (2). They obtained the symmetry reductions, group invariant solutions, and rich new exact solutions of the equation. Their solutions consist of elliptic functions in rational solutions, airy function solutions, polynomial solutions, trigonometric function solutions, hyperbolic function solutions, and elliptic periodic solutions. Nevertheless, some of their exact solutions are written in the integral forms of arbitrary functions which are not convenient to use.

(b) In [44], the authors employed the complex method to analytically solve (2). Instead of directly solving (2), they obtained the solutions of the transformed equation corresponding to the original problem. They acquired the meromorphic solutions of the transformed equation including the rational function solutions, the simply periodic solutions, i.e., hyperbolic cotangent function solutions, and the elliptic function solutions.

It is quite difficult to individually compare our obtained solutions with the solutions in the abovementioned references due to the generality of arbitrary functions in their solutions. Comparing in terms of mathematical structures between our solutions and the solutions from the previous works, the formulas of our solutions are written using the same functions as appearing in their results such as trigonometric, hyperbolic, rational, and elliptic functions. However, the number of our exact solutions especially obtained utilizing the Jacobi elliptic equation method are more than the number of ones generated in the previous literature. In addition, some of our solutions are distinct from those found in $[42,44]$ and have not been published elsewhere before. In summary, the two methods employed in the present paper are powerful, efficient, and reliable schemes in searching the exact traveling wave solutions for a wide range of NPDEs.

\section{Data Availability}

No data were used to support this study.

\section{Conflicts of Interest}

The authors declare that they have no conflicts of interest.

\section{Acknowledgments}

The authors would like to thank the referees for the valuable comments that helped us to improve the manuscript. The first author was funded by King Mongkut's University of Technology North Bangkok under contract no. KMUTNB61-PHD-014 and also partially supported by the Graduate College, King Mongkut's University of Technology North Bangkok. The second author was financially supported by the Faculty of Applied Science, King Mongkut's University of Technology North Bangkok under contract no. 6242106.

\section{References}

[1] M. Jaulent and I. Miodek, "Nonlinear evolution equations associated with enegry-dependent Schrödinger potentials," Letters in Mathematical Physics, vol. 1, no. 3, pp. 243-250, 1976.

[2] M. Jaulent, "Inverse scattering problems in absorbing media," Journal of Mathematical Physics, vol. 17, no. 7, pp. 1351-1360, 1976.

[3] J. Rana and S. Liao, "On time independent Schrödinger equations in quantum mechanics by the homotopy analysis method," Theoretical and Applied Mechanics Letters, vol. 9, no. 6, pp. 376-381, 2019.

[4] S. Sirisubtawee and S. Kaewta, "New modified Adomian decomposition recursion schemes for solving certain types of nonlinear fractional two-point boundary value problems," International Journal of Mathematics and Mathematical Sciences, vol. 2017, Article ID 5742965, 20 pages, 2017.

[5] S. Sirisubtawee, S. Koonprasert, and S. Kaewta, "Duan-Rach modified decomposition method for solving some types of nonlinear fractional multi-point boundary value problems," Far East Journal of Mathematical Sciences (FJMS), vol. 102, no. 10, pp. 2143-2176, 2017.

[6] M. Nadeem, F. Li, and H. Ahmad, "Modified laplace variational iteration method for solving fourth-order parabolic partial differential equation with variable coefficients," Computers \& Mathematics with Applications, vol. 78, no. 6, pp. 2052-2062, 2019.

[7] S. Owyed, M. Abdou, A.-H. Abdel-Aty, W. Alharbi, and R. Nekhili, "Numerical and approximate solutions for coupled 
time fractional nonlinear evolutions equations via reduced differential transform method," Chaos, Solitons \& Fractals, 2019.

[8] N. A. Kudryashov, "One method for finding exact solutions of nonlinear differential equations," Communications in Nonlinear Science and Numerical Simulation, vol. 17, no. 6, pp. 2248-2253, 2012.

[9] S. Tuluce Demiray, Y. Pandir, and H. Bulut, "Generalized Kudryashov method for time-fractional differential equations," Abstract and Applied Analysis, vol. 2014, Article ID 901540, 13 pages, 2014.

[10] E. Zayed, G. Moatimid, and A.-G. Al-Nowehy, "The generalized Kudryashov method and its applications for solving nonlinear PDEs in mathematical physics," Scientific Journal of Mathematics Research, vol. 5, no. 2, pp. 19-39, 2015.

[11] A. R. Seadawy, "Modulation instability analysis for the generalized derivative higher order nonlinear Schrödinger equation and its the bright and dark soliton solutions," Journal of Electromagnetic Waves and Applications, vol. 31, no. 14, pp. 1353-1362, 2017.

[12] H. Kheir, A. Jabbari, A. Yildirim, and A. Alomari, "He's semi-inverse method for soliton solutions of Boussinesq system," World Journal of Modelling and Simulation, vol. 9, pp. 3-13, 2013.

[13] R. T. Alqahtani, M. M. Babatin, and A. Biswas, "Bright optical solitons for Lakshmanan-Porsezian-Daniel model by semiinverse variational principle," Optik, vol. 154, pp. 109-114, 2018.

[14] J.-H. He and X.-H. Wu, "Exp-function method for nonlinear wave equations," Chaos, Solitons \& Fractals, vol. 30, no. 3, pp. 700-708, 2006.

[15] X.-H. B. Wu and J.-H. He, "Solitary solutions, periodic solutions and compacton-like solutions using the Exp-function method," Computers \& Mathematics with Applications, vol. 54, no. 7-8, pp. 966-986, 2007.

[16] A. Akbulut and M. Kaplan, "Auxiliary equation method for time-fractional differential equations with conformable derivative," Computers \& Mathematics with Applications, vol. 75, no. 3, pp. 876-882, 2018.

[17] B.-Q. Li and Y.-L. Ma, "Loop-like periodic waves and solitons to the Kraenkel-Manna-Merle system in ferrites," Journal of Electromagnetic Waves and Applications, vol. 32, no. 10, pp. 1275-1286, 2018.

[18] Q. Zhou, M. Ekici, A. Sonmezoglu, M. Mirzazadeh, and M. Eslami, "Optical solitons with Biswas-Milovic equation by extended trial equation method," Nonlinear Dynamics, vol. 84, no. 4, pp. 1883-1900, 2016.

[19] M. Ekici, M. Mirzazadeh, A. Sonmezoglu et al., "Optical solitons with anti-cubic nonlinearity by extended trial equation method," Optik, vol. 136, pp. 368-373, 2017.

[20] A. R. Seadawy, "Solitary wave solutions of two-dimensional nonlinear Kadomtsev-Petviashvili dynamic equation in dustacoustic plasmas," Pramana, vol. 89, no. 3, p. 49, 2017.

[21] A.-M. Wazwaz, "A sine-cosine method for handlingnonlinear wave equations," Mathematical and Computer Modelling, vol. 40, no. 5-6, pp. 499-508, 2004.

[22] M. Alquran and K. Al-Khaled, "The tanh and sine-cosine methods for higher order equations of Korteweg-de Vries type," Physica Scripta, vol. 84, no. 2, Article ID 025010, 2011.

[23] A.-M. Wazwaz, "The tanh-coth method for solitons and kink solutions for nonlinear parabolic equations," Applied Mathematics and Computation, vol. 188, no. 2, pp. 1467-1475, 2007.

[24] A.-M. Wazwaz, "The tanh-coth method for new compactons and solitons solutions for the $K(n, n)$ and the $K(n+1, n+1)$ equations," Applied Mathematics and Computation, vol. 188, no. 2, pp. 1930-1940, 2007.

[25] A. R. Seadawy, "Ion acoustic solitary wave solutions of twodimensional nonlinear Kadomtsev-Petviashvili-Burgers equation in quantum plasma," Mathematical Methods in the Applied Sciences, vol. 40, no. 5, pp. 1598-1607, 2017.

[26] A. Korkmaz, O. E. Hepson, K. Hosseini, H. Rezazadeh, and M. Eslami, "Sine-Gordon expansion method for exact solutions to conformable time fractional equations in RLWclass," Journal of King Saud University-Science, vol. 32, no. 1, pp. $567-574,2018$.

[27] H. Bulut, T. A. Sulaiman, and B. Demirdag, "Dynamics of soliton solutions in the chiral nonlinear Schrödinger equations," Nonlinear Dynamics, vol. 91, no. 3, pp. 1985-1991, 2018.

[28] H. M. Baskonus, T. A. Sulaiman, and H. Bulut, "Novel complex and hyperbolic forms to the strain wave equation in microstructured solids," Optical and Quantum Electronics, vol. 50, no. 1, p. 14, 2018.

[29] M. Wang, X. Li, and J. Zhang, "The ()-expansion method and travelling wave solutions of nonlinear evolution equations in mathematical physics," Physics Letters A, vol. 372, no. 4, pp. 417-423, 2008.

[30] A. Bekir, "Application of the -expansion method for nonlinear evolution equations," Physics Letters A, vol. 372, no. 19, pp. 3400-3406, 2008.

[31] E. Zayed and K. A. Gepreel, "The $\left(G^{\prime} / G\right)$-expansion method for finding traveling wave solutions of nonlinear partial differential equations in mathematical physics," Journal of Mathematical Physics, vol. 50, no. 1, Article ID 013502, 2009.

[32] Y. Li, X. Liu, and X. Xin, "Explicit solutions, conservation laws of the extended $(2+1)$-dimensional Jaulent-Miodek equation," 2015, https://arxiv.org/abs/1512.09196.

[33] M. Hong-Cai, D. Ai-Ping, and Y. Yao-Dong, "Lie symmetry group of $(2+1)$-dimensional Jaulent-Miodek equation," Thermal Science, vol. 18, no. 5, 2014.

[34] A.-M. Wazwaz, "The tanh-coth and the sech methods for exact solutions of the Jaulent-Miodek equation," Physics Letters A, vol. 366, no. 1-2, pp. 85-90, 2007.

[35] J.-H. He and L.-N. Zhang, "Generalized solitary solution and compacton-like solution of the Jaulent-Miodek equations using the Exp-function method," Physics Letters A, vol. 372, no. 7, pp. 1044-1047, 2008.

[36] J.-M. Zhu, Z. M. Lu, and L. Liu, "Doubly periodic wave solutions of jaulent-miodek equations using variational iteration method combined with jacobian-function method," Communications in Theoretical Physics, vol. 49, no. 6, pp. 14031406, 2008.

[37] A.-M. Wazwaz, "Multiple kink solutions and multiple singular kink solutions for -dimensional nonlinear models generated by the Jaulent-Miodek hierarchy," Physics Letters A, vol. 373, no. 21, pp. 1844-1846, 2009.

[38] E. M. Zayed and H. M. Rahman, “The extended tanh-method for finding traveling wave solutions of nonlinear evolution equations," Applied Mathematics E-Notes, vol. 10, pp. 235245, 2010.

[39] H.-L. Lü, X.-Q. Liu, and L. Niu, "A generalized-expansion method and its applications to nonlinear evolution equations," Applied Mathematics and Computation, vol. 215, no. 11, pp. 3811-3816, 2010.

[40] W. M. Taha and M. S. M. Noorani, "Exact solutions of equation generated by the Jaulent-Miodek hierarchy by $\left(G^{\prime} / G\right)$-expansion method," Mathematical Problems in Engineering, vol. 2013, Article ID 392830, 7 pages, 2013. 
[41] A.-M. Wazwaz, "Multiple soliton solutions for some $(3+1)$ dimensional nonlinear models generated by the JaulentMiodek hierarchy," Applied Mathematics Letters, vol. 25, no. 11, pp. 1936-1940, 2012.

[42] Y.-Y. Zhang, X.-Q. Liu, and G.-W. Wang, "Symmetry reductions and exact solutions of the $(2+1)$-dimensional Jaulent-Miodek equation," Applied Mathematics and Computation, vol. 219, no. 3, pp. 911-916, 2012.

[43] M. Matinfar, M. Eslami, and S. Roshandel, "The first integral method to study the $(2+1)$-dimensional Jaulent-Miodek equations," Pramana, vol. 85, no. 4, pp. 593-603, 2015.

[44] Y. Gu, B. Deng, and J. Lin, "Exact traveling wave solutions to the $(2+1)$-dimensional Jaulent-Miodek equation," Advances in Mathematical Physics, vol. 2018, Article ID 5971646, 9 pages, 2018.

[45] R. Sadat and M. Kassem, "Explicit solutions for the $(2+1)$ dimensional jaulent-miodek equation using the integrating factors method in an unbounded domain," Mathematical and Computational Applications, vol. 23, no. 1, p. 15, 2018.

[46] S. Sirisubtawee and S. Koonprasert, "Exact traveling wave solutions of certain nonlinear partial differential equations using the $\left(G^{\prime} / G^{2}\right)$-expansion method," Advances in Mathematical Physics, vol. 2018, Article ID 7628651, 15 pages, 2018.

[47] S. T. Mohyud-Din and S. Bibi, "Exact solutions for nonlinear fractional differential equations using $G^{\prime} G 2$-expansion method," Alexandria Engineering Journal, vol. 57, no. 2, pp. 1003-1008, 2018.

[48] J. Chen and H. Chen, "The $\left(G^{\prime} / G^{2}\right)$-expansion method and its application to coupled nonlinear Klein-Gordon equation," Journal of South China Normal University (Natural Science Edition), vol. 2, 2012.

[49] E. M. E. Zayed and A.-G. Al-Nowehy, "Exact solutions for the perturbed nonlinear Schrödinger equation with power law nonlinearity and Hamiltonian perturbed terms," Optik, vol. 139, pp. 123-144, 2017.

[50] E. M. Zayed, Y. A. Amer, and R. M. Shohib, "The Jacobi elliptic function expansion method and its applications for solving the higher order dispersive nonlinear Schrödinger equation," Nonlinearity, vol. 37, no. 38, p. 38, 2014.

[51] Q. Zhou, D. Yao, and F. Chen, "Analytical study of optical solitons in media with Kerr and parabolic-law nonlinearities," Journal of Modern Optics, vol. 60, no. 19, pp. 1652-1657, 2013.

[52] B. Zheng and Q. Feng, "The jacobi elliptic equation method for solving fractional partial differential equations," Abstract and Applied Analysis, vol. 2014, Article ID 249071, 9 pages, 2014. 